\title{
Anticorrelation between temperature and fluctuations in moderately damped Josephson junctions
}

\author{
V.M.Krasnov and T.Golod \\ Department of Physics, Stockholm University, Albanova University Center, SE-10691 Stockholm, Sweden \\ T.Bauch and P.Delsing \\ Department of Microtechnology and Nanoscience, \\ Chalmers University of Technology, SE-41296 Göteborg, Sweden
}

\begin{abstract}
We study the influence of dissipation on the switching current statistics of moderately damped Josephson junctions. Different types of both low- and high- $T_{c}$ junctions with controlled damping are studied. The damping parameter of the junctions is tuned in a wide range by changing temperature, magnetic field, gate voltage, introducing a ferromagnetic layer or in-situ capacitive shunting. A paradoxical collapse of switching current fluctuations occurs with increasing $T$ in all studied junctions. The phenomenon critically depends on dissipation in the junction and is explained by interplay of two counteracting consequences of thermal fluctuations, which on the one hand assist in premature switching into the resistive state and on the other hand help in retrapping back to the superconducting state. This is one of the rare examples of anticorrelation between temperature and fluctuation amplitude of a physically measurable quantity.
\end{abstract}

PACS numbers: $74.40 .+\mathrm{k}, 74.50 .+\mathrm{r}, 74.45 .+\mathrm{c}, 74.72 . \mathrm{Hs}$

\section{INTRODUCTION}

Temperature is a measure of the energy of thermal fluctuations. For example, it is well known that noise in electronic components or Brownian motion of small particles increase with temperature. But does the amplitude of fluctuations of physical properties always increase with $T$ ? Recently a spectacular exception from this rule was reported almost simultaneously by three groups of researchers [1, 2, 3]. It was observed that fluctuations of the bias current required for switching of a Josephson junction (JJ) from the superconducting $(S)$ to the resistive $(R)$ state may suddenly collapse (drastically decrease) at elevated $T$. It was suggested that the paradoxical behavior is caused by the fact that temperature does not only provide energy for excitation of a system from the equilibrium state but also enhance the rate of relaxation back to the equilibrium. The latter strongly depends on the damping in the system and under certain circumstances can reverse the correlation between fluctuations and temperature.

Dissipation plays a crucial role in decay of metastable states, which determines dynamics of various physical and chemical processes [4, 5]. Switching between $S$ and $R$ states in JJ's is one of the best studied examples of such a decay. The influence of dissipation on the switching statistics of JJ's has been intensively studied both theoretically [4, 6, 7, 8, 9] and experimentally [10, 11, 12, 13, 14, 15]. The role of dissipation in decoherence of quantum systems has been widely discussed [16] and has recently become an important issue for quantum computing. JJ's are used in several different ways in qubit implementations. For example, current biased JJ's are employed in phase qubits [17], where the dissipation affects relaxation and decoherence in the qubits. Furthermore, switching of JJ's is also used for read-out of both flux [18] and charge-phase [19] qubits.

Here we present an extensive study of dissipation effects on the phase dynamics in moderately damped JJ's. For this purpose we prepared several types of junctions with well controlled and tunable damping parameters. In particular, we study low ohmic Nb-Pt-Nb Superconductor-Normal metal-Superconductor (SNS) junctions, Nb-CuNi-Nb Superconductor-FerromagnetSuperconductor (SFS) junctions with a diluted feromagnetic alloys, and Nb-InAs-Nb Superconductor - two dimensional electron gas - Superconductor (S-2DEG-S) junctions, as well as $\mathrm{Bi}_{2} \mathrm{Sr}_{2} \mathrm{CaCu}_{2} \mathrm{O}_{8+\delta}$ (Bi-2212) high$T_{c}$ (HTSC) intrinsic JJ's. The influence of dissipation on thermal and quantum fluctuations is studied by tuning damping parameters of the junctions by temperature, magnetic field, gate voltage, introducing a ferromagnetic layer or capacitive shunting. The paradoxical collapse of switching current fluctuations with increasing $T$ was observed in all cases. It is shown that the collapse temperature critically depends on dissipation in the junction. The phenomenon is explained by interplay of two conflicting consequences of thermal fluctuations, which on one hand assist in premature switching to the resistive state and on the other hand help in retrapping back to the superconducting state. The conclusions are supported by analytical calculations and numerical simulations, which are in quantitative agreement with new experimental data presented here as well as with those 
reported in Refs. [1, 2, 3].

The paper is organized as follows. In section II we summarize the results of the Resistively and Capacitively Shunted Junction (RCSJ) model, required for the analysis of switching statistics in moderately damped JJ's. In Section III we characterize the JJ's studied in this work and describe the experimental techniques. In Sec. IV we analyze the magnetic field modulation of the switching and retrapping currents. This helps to understand the origin of hysteresis in the current-voltage characteristics (IVC's) and to estimate the damping parameters of our JJ's. In Sec. V the main experimental results on the switching current statistics are presented. It is shown that the collapse of thermal activation (TA) occurs in all moderately damped JJ's and that the macroscopic quantum tunneling (MQT) phenomena persists even in strongly damped SNS-type JJ's. Finally, in Sec. VI we discuss the mechanism of the paradoxical collapse of TA in moderately damped JJ's and present numeric and analytic calculations, which clarify the phase dynamics in the collapsed state and support our conclusions.

\section{GENERAL RELATIONS}

The damping parameter of JJ's within the RCSJ model is characterized by the inverse value of the quality factor at zero bias current, $Q_{0}$. For junctions with sinusoidal current-phase relation (CPR) it is given by:

$$
Q_{0}=\omega_{p 0} R C=\sqrt{2 e I_{c 0} R^{2} C / \hbar} .
$$

Here $\omega_{p 0}=\left(2 e I_{c 0} / \hbar C\right)^{1 / 2}$ is the Josephson plasma frequency at zero bias, $R$ and $C$ are the junction resistance and capacitance, respectively, and $I_{c 0}$ is the fluctuationfree critical current. Determination of $Q_{0}$ is not trivial: $I_{c 0}$ must be obtained by measurement and extrapolation of the switching current statistics [11]; the effective capacitance is not equal to the geometric one because leads form a sort of transmission line with finite inductance; for Superconductor-Insulator-Superconductor (SIS) tunnel junctions the effective resistance is ill-defined. Conflicting reports exists on what determines the effective damping in SIS junctions: the normal resistance [10], the high frequency impedance of circuitry [11], the quasiparticle resistance [12], or the up-transformed lead impedance [20]. Furthermore, the resistance of SIS junctions is frequency and bias dependent due to the strong nonlinearity of the IVC's.

At finite bias current, the quality factor, $Q(I)$, is given by the same Eq.(1) with $\omega_{p 0}$ replaced by the Josephson plasma frequency at finite bias, which for junctions with the sinusoidal CPR is: $\omega_{p}(I)=\omega_{p 0}\left(1-\left(I / I_{c 0}\right)^{2}\right)^{1 / 4}$. This means that the quality factor is bias dependent, $Q(I)=Q_{0}\left(1-\left(I / I_{c 0}\right)^{2}\right)^{1 / 4}$. For SIS junctions $Q(I)$ can
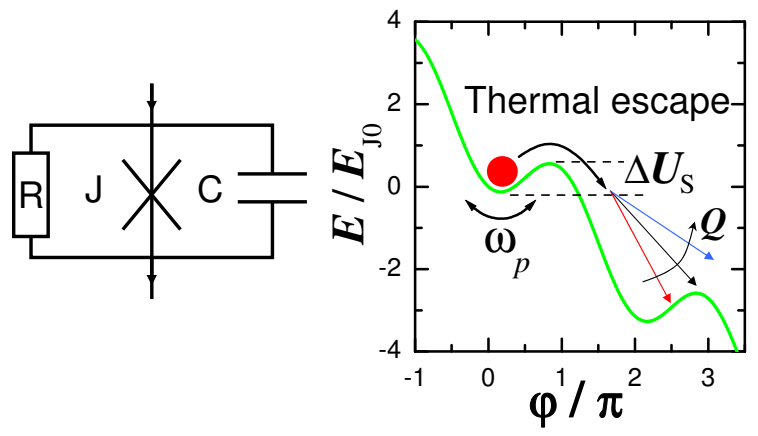

FIG. 1: (Color online). Left: the equivalent circuit of the RCSJ model. Right: the mechanical analog of the RCSJ model: the tilted wash board potential in the energy-phase space for $I=0.5 I_{c 0}$. Arrows indicate three possible particle trajectories after thermal escape for different quality factors. For the lowest $Q$ the particle get's retrapped in the next potential well, while for highest $Q$ it will continue to roll down the potential, leading to switching of the JJ from the superconducting to the resistive state.

be further reduced due to frequency dependence of $R$. Therefore, the phase dynamics may change from underdamped, $Q \gtrsim 1$, to overdamped $Q \lesssim 1$ as $I \rightarrow I_{c 0}$.

The electrodynamics of JJ's is equivalent to motion of a particle in a tilted wash-board potential formed by superposition of the periodic Josephson potential and the work done by the current source (the tilt), as shown in Fig.1. The particle can escape from the potential well as a result of MQT or TA process. At low damping the escaped particle will roll down the potential (switch to the $R$-state). However, if dissipation exceeds the work done by the current source it will be retrapped in subsequent wells and return to the $S$-state, as shown in Fig. 1.

The TA escape rate from $S$ to $R$ state is determined by an Arrhenius law, and for moderate and high damping cases it is given by [7]:

$$
\Gamma_{T A}=a_{t} \frac{\omega_{p}(I)}{2 \pi} \exp \left[-\frac{\Delta U_{S}(I)}{k_{B} T}\right] .
$$

Here $\Delta U_{S}$ is the potential barrier for $S-R$ switching, see Fig. 1, which for the sinusoidal CPR is

$$
\Delta U_{S} \simeq(4 \sqrt{2} / 3) E_{J 0}\left[1-I / I_{c 0}\right]^{3 / 2},
$$

where $E_{J 0}=(\hbar / 2 e) I_{c 0}$ is the Josephson energy. Damping enters only into the prefactor of $\Gamma_{T A}$ :

$$
a_{t}=\left(1+1 / 4 Q^{2}\right)^{1 / 2}-1 / 2 Q .
$$

The MQT escape rate can be written in notations of Ref. 8] as

$$
\Gamma_{M Q T}=\gamma(T) \frac{\omega_{p}}{2 \pi}\left[\frac{\Delta U_{S}}{\hbar \omega_{p}}\right]^{1 / 2} \chi(Q) \exp \left[-\frac{\Delta U_{S}}{\hbar \omega_{p}} s(Q)\right] .
$$


Here $\gamma(T)$ is the thermal correction with the characteristic parabolic dependence $\ln \gamma \propto T^{2}$; and in the case of strong damping $\chi(Q) \simeq 2 \pi \sqrt{3} Q^{7 / 2}\left[1-Q^{2}(8 \ln (2 Q)-\right.$ 4.428) $]$ and $s(Q) \simeq 3 \pi\left[Q+Q^{-1}\right]$.

The MQT rate can be strongly affected by dissipation since the damping dependent factor $s(Q)$ appears under the exponent in Eq.(5). Qualitatively this is due to smearing of quantum levels in the wash-board potential with decreasing $Q$. Indeed, the spacing between levels is $\sim \hbar \omega_{p}$, while the level width is $\sim \hbar / R C$. From Eq.(1) it follows that for $Q<1$, the width becomes larger than the separation between levels, which leads to suppression of the MQT.

In the moderate damping case the crossover between MQT and TA occurs at [7]

$$
T_{M Q T}(Q)=\frac{\hbar \omega_{p}}{2 \pi k_{B}}\left[\left(1+\frac{1}{4 Q^{2}}\right)^{1 / 2}-\frac{1}{2 Q}\right] .
$$

From Eq.(6) it follows that the MQT-TA crossover temperature decreases with $Q$ due to general suppression of the MQT, as discussed above.

An analytic expression for the retrapping rate from the $R$ to the $S$ state is known only for strongly underdamped JJ's $Q_{0} \gg 1[\underline{6}]$ :

$$
\begin{array}{r}
\Gamma_{R}=\omega_{p 0} \frac{I-I_{R 0}}{I_{c 0}} \sqrt{\frac{E_{J 0}}{2 \pi k_{B} T}} \exp \left[-\frac{\Delta U_{R}(I)}{k_{B} T}\right], \\
\Delta U_{R} \simeq \frac{E_{J 0} Q_{0}^{2}}{2}\left[\frac{I-I_{R 0}}{I_{c 0}}\right]^{2} .
\end{array}
$$

Here $I_{R 0}$ is the fluctuation-free retrapping current and $\Delta U_{R}$ the retrapping (dissipation) barrier. From Eq.(8) it is seen that retrapping, unlike escape, depends strongly on damping [15], because $Q_{0}^{2}$ appears under the exponent in Eq.(7). For underdamped JJ's the $I_{R 0}$ is given by

$$
I_{R 0} \simeq \frac{4 I_{c 0}}{\pi Q_{0}}
$$

This expression is valid for $Q_{0} \geqslant 3$. For smaller $Q_{0}$

$I_{R 0} / I_{c 0} \simeq 1.27299-0.31102 Q_{0}-0.02965 Q_{0}^{2}+0.01306 Q_{0}^{3}$.

Eq.(10) was obtained from interpolation of the numerically simulated IVC's within the RCSJ model [21]. This expression is valid for $0.84 \lesssim Q_{0}<3$. For $Q_{0} \lesssim 0.84$, the IVC's are non-hysteretic, i.e., $I_{R 0}=I_{c 0}$.

\section{Switching statistics}

For a given switching rate $\Gamma_{01}$ from the initial state 0 to state 1 , the probability of switching within the infinitesimal time interval $\delta t$ is given by $\mathbb{P}_{01}(\delta t)=\delta t \Gamma_{01}$. The probability of staying in the 0 state is $\mathbb{P}_{0}(\delta t)=1-\delta t \Gamma_{01}$.
The probability of staying in the 0 state within a finite time interval $t$ is then given by the conditional probability of not switching during all sub-intervals $\delta t$ : $\mathbb{P}_{0}(t)=\lim _{\delta t \rightarrow 0}\left(1-\delta t \Gamma_{01}\right)^{t / \delta t}=\exp \left[-\Gamma_{01} t\right]$.

Measurements of switching statistics in JJ's are typically performed by ramping the bias current at constant rate $d I / d t$. Since the switching rate $\Gamma_{01}(I)$ is bias dependent, the probability of not switching until current $I$ can be written as

$$
\mathbb{P}_{0}(I)=\exp \left[-\frac{1}{d I / d t} \int_{0}^{I} \Gamma_{01}(I) d I\right]
$$

Alternatively, it can be written as

$$
\mathbb{P}_{0}(I)=1-\int_{0}^{I} P_{01}(I) d I,
$$

where $P_{01}(I)$ is the probability density of switching from 0 to 1 state. By definition $P_{01}(I) \delta I$ is the probability of switching in the bias interval from $I$ to $I+\delta I$. The probability density is one of the most important characteristics of switching statistics because it directly corresponds to experimentally measured switching current histograms.

Differentiating Eqs. (1112) with respect to $I$ we obtain:

$$
P_{01}=-\frac{\mathbb{P}_{0}(I)}{d I}=\frac{\Gamma_{01}(I)}{d I / d t}\left(1-\int_{0}^{I} P_{01}(I) d I\right) .
$$

This equation has a clear physical meaning: the probability of measuring the switching event in the current interval from $I$ to $I+\delta I$ is the conditional probability of switching during the ramping time $\delta t=\delta I /(d I / d t)$ (first term), and the probability that the system has not already switched before (second term). The recurrent equation (13) is easily solved numerically and couples the switching probability density $P_{01}$ to the switching rate $\Gamma_{01}$.

The probability density for switching from $S$ to $R$ state follows directly from Eq.(13), where 0 and 1 are $S$ and $R$ states respectively. To obtain the probability density of retrapping, $P_{R}$, from $R$ to $S$-state we should take into account that current is now ramped downwards to zero and that $P_{R}=0$ at $I \geq I_{c 0}$ :

$$
P_{R}(I)=\frac{\Gamma_{R}(I)}{|d I / d t|}\left[1-\int_{I}^{I_{c 0}} P_{R}(I) d I\right] .
$$

The probability of remaining in the $R$ state and not being retraped until current $I$ is

$$
\mathbb{P}_{n R}(I)=1-\int_{I}^{I_{c 0}} P_{R}(I) d I
$$


TABLE I: Summary of the studied junctions.

\begin{tabular}{|c|c|c|c|c|c|}
\hline Junction & Geometry & $I_{c 0}(\mu A)$ & $R(\Omega)$ & $\overline{T^{*}(\mathrm{~K})}$ & $Q_{0}\left(T^{*}\right)$ \\
\hline SFS & $\mathrm{Nb} / \mathrm{CuNi}$ & & & & \\
\hline$\# 1 \mathrm{a}$ & $70 / 50 \mathrm{~nm}$ & 770 & 0.24 & - & - \\
\hline$\# 2 \mathrm{a}$ & $25 / 50 \mathrm{~nm}$ & 178 & 0.21 & $0.2^{a}$ & $1.0^{a}$ \\
\hline$\# 2 \mathrm{~b}$ & $25 / 50 \mathrm{~nm}$ & 34 & 0.26 & - & $\gtrsim 0.84$ \\
\hline S-2DEG-S & 2DEG $w \times L$ & & & & \\
\hline$\# 1 \mathrm{a}$ & $10 \mu m \times 500 \mathrm{~nm}$ & 2.5 & 36 & - & $\sim 0.84$ \\
\hline$\# 2 \mathrm{~b}$ & $40 \mu m \times 400 n m$ & 37 & 7.5 & 0.8 & 1.63 \\
\hline$\# 3 \mathrm{a}$ & $10 \mu m \times 500 \mathrm{~nm}$ & 2.3 & 38.1 & - & 0.88 \\
\hline$\# 3 b$ & $40 \mu m \times 500 n m$ & 7.3 & 11.4 & 0.1 & 0.95 \\
\hline C-shunted & & & & & \\
\hline$\# 3 \mathrm{a}$ & $10 \mu m \times 500 n m$ & 2.5 & 34 & 0.45 & 2.4 \\
\hline$\# 3 \mathrm{~b}$ & $40 \mu m \times 500 n m$ & 7.4 & 10.3 & $>1$ & $>3$ \\
\hline SNS & $170 \times 88 \mathrm{~nm}^{2}$ & 262 & 0.6 & - & $<0.84$ \\
\hline $\mathrm{Bi}-2212$ & $4 \times 2.5 \mu m^{2}$ & 125 & $\sim 40$ & 75 & 5.6 \\
\hline
\end{tabular}

Thermal fluctuations lead to premature switching and retrapping. This means that fluctuations tend to decrease the switching current $I_{S}$ with respect to $I_{c 0}$, but increase the retrapping current, $I_{R}$, with respect to $I_{R 0}$. Therefore, thermal fluctuations help in returning the JJ from $R$ to $S$ state. One should also keep in mind that since retrapping critically depends on damping and damping depends on bias, retrapping can become prominent at the switching current even for junctions which are underdamped at zero bias.

\section{SAMPLES}

Analysis of dissipation effects on phase dynamics requires junctions with well defined and controlled damping parameters. Ideally such junctions should be RCSJ-type with bias independent $R$; and have $R$ much smaller than the high frequency impedance of the circuitry $Z_{0} \sim 100 \Omega$. Previous studies of fluctuation phenomena in JJ's were performed predominantly on underdamped, $Q_{0} \gg 1$, SIS tunnel junctions, which are not described by the simple RCSJ model with constant, $Q_{0}$, both due to strongly non-linear IVC's and considerable shunting by the high frequency impedance 9]. This ambiguity does not exists for SNS junctions, which are well described by RCSJ model with constant $R$, typically much smaller than $Z_{0}$.

Although the quality factor of SNS junctions is expected to be constant, verification of this as well as exact evaluation of $Q_{0}$ is non trivial. Parameters $I_{c 0}$ and $C$ still need to be independently defined. Furthermore, Eq.(1) is valid only for junctions with sinusoidal CPR. The CPR in SNS junctions deviates from sinusoidal [22], which changes the plasma frequency $\omega_{p 0}$ and thus affects the effective $C$ entering Eq.(1). The effective $C$ is no longer equal to the real, explicitly measurable junction capacitance. Therefore, quantitative analysis of dissipation effects requires a possibility of tuning the quality

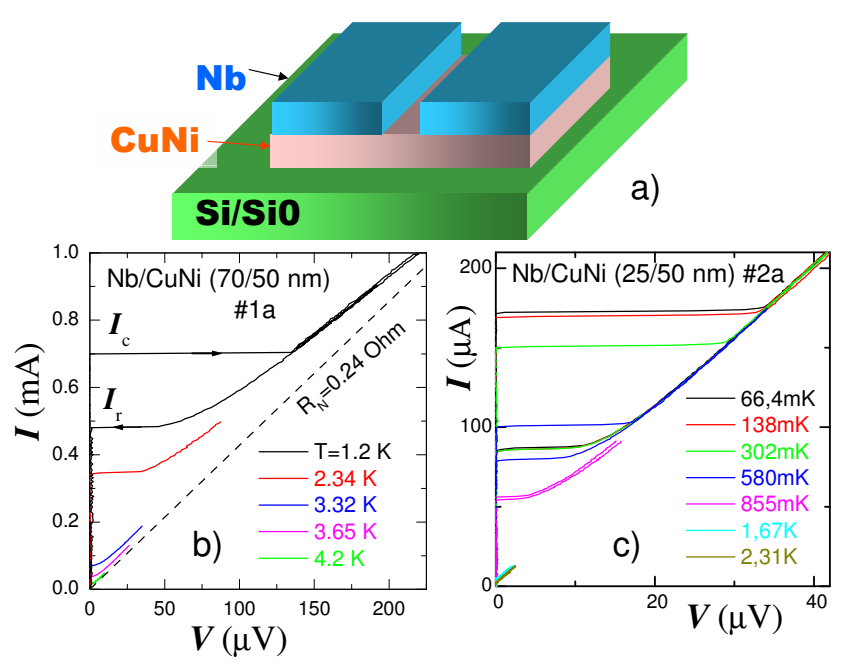

FIG. 2: (Color online). a) Sketch of the planar Nb-CuNi$\mathrm{Nb}$ junction. The junctions were made by cutting $\mathrm{Nb} / \mathrm{CuNi}$ bilayers by FIB. Panels b) and c) show $I-V$ characteristics at different $T$ for junctions made from $70 / 50 \mathrm{~nm}$ and 25/50 $\mathrm{nm}$ thick $\mathrm{Nb} / \mathrm{CuNi}$ bilayers, respectively. The IVC's of both junctions are RCSJ-like and exhibit hysteresis at low $T$.

factor by at least as many independent parameters as the amount of unknown variables in Eq.(1).

In this work we focus on the analysis of phase dynamics in low ohmic SNS-type junctions with moderate damping $1 \lesssim Q_{0}<10$. Emphasis was made on the ability to control and tune the damping parameter of the junctions. Below we describe five different ways used for tuning and verification of the quality factor of our junctions: together with conventional ways of tuning $I_{c 0}$ by applying the magnetic field or changing temperature, we were also able to tune $I_{c 0}$ by applying gate voltage, adding a ferromagnetic material into the junction barrier and by tuning $C$ by in-situ capacitive shunting.

The parameters of the studied junctions are summarized in Table 1.

\section{Planar SFS (Nb-CuNi-Nb) junctions}

Planar Nb-CuNi-Nb junctions were made by cutting a small $\mathrm{Nb} / \mathrm{Cu}_{0.47} \mathrm{Ni}_{0.53}$ bilayer bridge by a Focused Ion Beam (FIB) 23]. A sketch of the SFS junction is shown in Fig.2 a). We made junctions from two types of $\mathrm{Nb} / \mathrm{CuNi}$ bilayers with either 70 or $25 \mathrm{~nm}$ thick $\mathrm{Nb}$ layers. The thickness of the $\mathrm{CuNi}$ layer was always $50 \mathrm{~nm}$. In-plane dimensions of the JJ's, presented here, were the same. Details of sample fabrication and characterization can be found elsewhere 24].

Fig. 2 b) and c) show the IVC's at different $T$ for planar SFS junctions made from $70 / 50 \mathrm{~nm}$ and $25 / 50$ $\mathrm{nm} \mathrm{Nb} / \mathrm{CuNi}$ bilayers, respectively. From Fig. 2 it is seen that the IVC's are consistent with the RCSJ model 


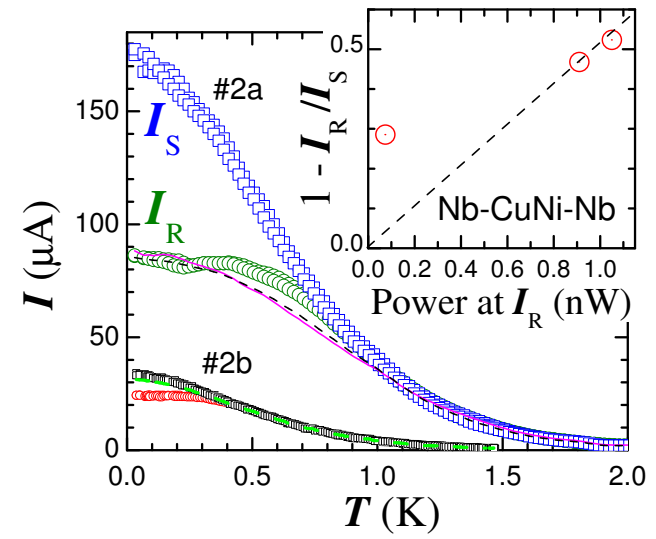

FIG. 3: (Color online). Temperature dependence of the switching and retrapping currents for two SFS junctions made from the same $25 / 50 \mathrm{~nm} \mathrm{Nb} / \mathrm{CuNi}$ bilayer at $H=0$. It is seen that the hysteresis $I_{S}>I_{R}$ exists at low $T$ for both JJ's. Solid and dashed lines represent $I_{R}(T)$ calculated within the RCSJ and self-heating models, respectively. The inset shows the size of the hysteresis $1-I_{R} / I_{S}$ at the lowest $T$ as a function of the dissipated power at $I=I_{R}$. Note that the hysteresis is not proportional to the power at retrapping.

with constant $R$. Since $R \ll Z_{0} \sim 100 \Omega$, shunting by the circuitry impedance is negligible also at high frequencies.

The critical current depends very strongly on the depth of the FIB cut. By varying the depth of the cut we were able to fabricate JJ's with three orders of magnitude difference in $I_{c 0}$ 24]. To the contrary, the resistance of junctions remained almost unchanged $R \sim 0.25 \Omega$ as seen from IVC's in Fig. 2. Since the in-plane geometries of the junctions are the same, $C$ and the thermal conductances of the junctions are also similar. Therefore, by changing the depth of the FIB cut we could vary in a wide range the $Q_{0}$ of the junctions by solely affecting $I_{c 0}$ and leaving all other parameters intact.

Fig. 3 shows $T$-dependencies of switching and retrapping currents for two JJ's on the same chip. Development of the hysteresis, $I_{S}>I_{R}$, with $T$ is seen. The JJ's had good uniformity of the critical current, as follows from the clear Fraunhofer modulation of the critical current as a function of magnetic field, shown in Fig. 4.

\section{Nano-sculptured SNS (Nb-Pt-Nb) junctions}

Nano-scale SNS junctions were made from $\mathrm{Nb}-\mathrm{Pt}-\mathrm{Nb}$ trilayers [25] by three-dimensional FIB sculpturing. The thicknesses of bottom and top Nb layers were 225 and $350 \mathrm{~nm}$, respectively. The thickness of $\mathrm{Pt}$ was $30 \mathrm{~nm}$. A sketch of the junction is shown in the inset in Fig. 5 . Nano-fabrication was required both for increasing $R$ and decreasing $I_{c 0}$ to easily measurable values.

The main panel in Fig. 5 shows a set of IVC's at $T=3.2 K$ for a Nb-Pt-Nb JJ $\left(170 \times 88 \mathrm{~nm}^{2}\right)$ at differ-

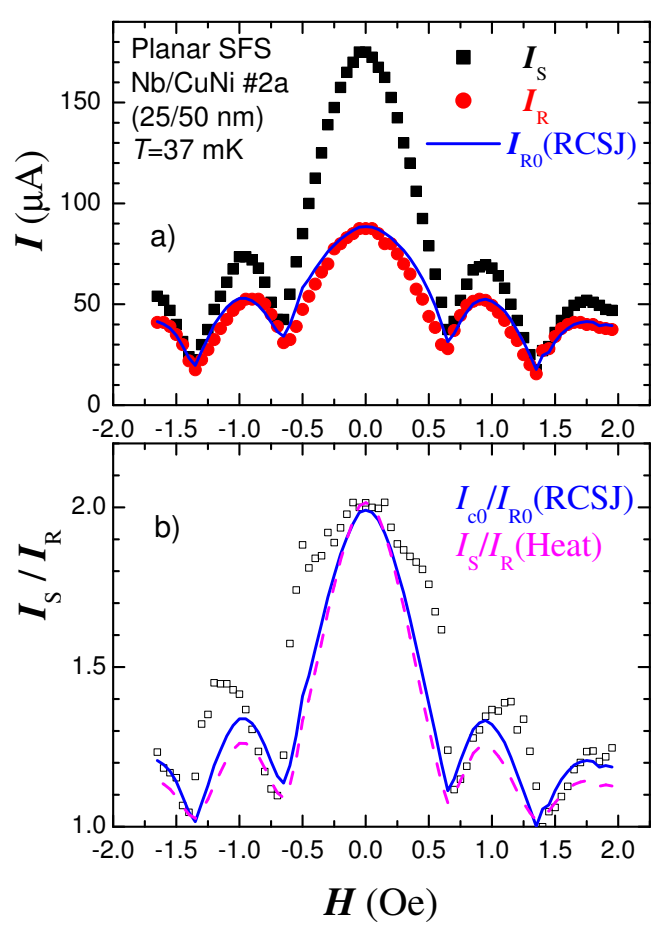

FIG. 4: (Color online). a) Magnetic field dependence of the switching and retrapping currents for the planar SFS junction $\# 2$ a at $T=37 \mathrm{mK}$. The solid line is the calculated $I_{R 0}(H)$ within the RCSJ model for $Q_{0}(H=0)=2.55$. b) $I_{S} / I_{R}$ vs $H$ for the same junction. The solid line is the $I_{c 0} / I_{R 0}$ within the RCSJ model for $Q_{0}(H=0)=2.55$. The dashed line represents $I_{c 0} / I_{R}$ calculated for the case when the hysteresis is caused solely by self-heating (see sec.IV).

ent magnetic fields along the long side of the JJ. Strong modulation of the critical current is seen. The IVC's are well described by the RCSJ model with constant $R \simeq 0.6 \Omega \ll Z_{0}$.

Some JJ's were anodized to remove possible shorts caused by redeposition of $\mathrm{Nb}$ during FIB etching, and to further decrease the junction area. The absence of shorts and the uniformity of junctions was confirmed by clear Fraunhofer modulation of $I_{c}(H)$, shown in Fig. 6. Details of the junction fabrication and characterization were described in Ref. [26] and will be published elsewhere.

\section{S-2DEG-S junctions}

S-2DEG-S junctions with planar geometry were formed by two $\mathrm{Nb}$ electrodes connected via the 2DEG (InAs) [27]. Properties $\left(I_{c 0}\right.$ and $\left.R\right)$ of the JJ's depend on the width of Nb-electrodes (either 10, or $40 \mu \mathrm{m}$ ), the length of the 2DEG (either 400 or $500 \mathrm{~nm}$ ) and the transparency of the contact between the $\mathrm{Nb}$ and the 2DEG [29]. A narrow gate electrode was made on top of the 2DEG, forming a Josephson field-effect transistor [28]. This provides a unique opportunity to tune properties of the JJ's by ap- 


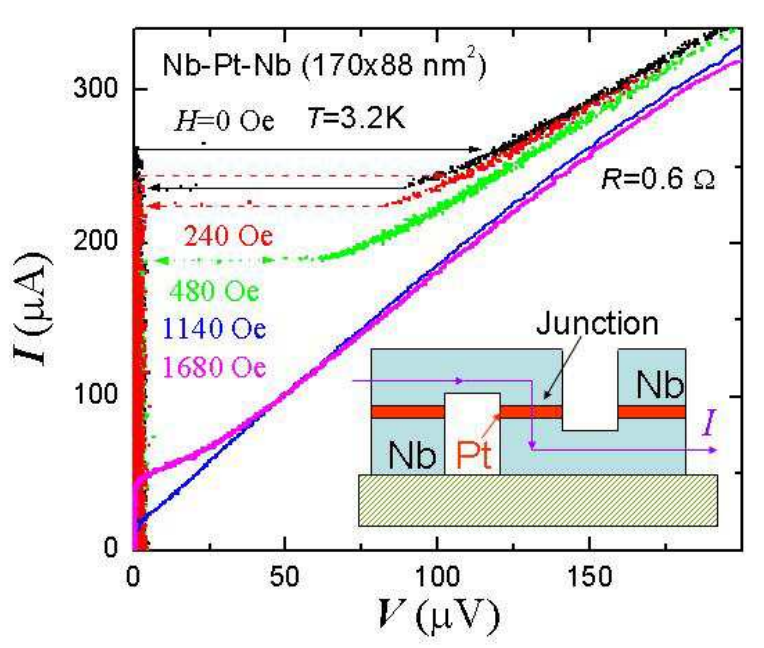

FIG. 5: (Color online). $I-V$ characteristics at different $H$ for a nano-sculptured SNS junctions at $T=3.2 \mathrm{~K}$. IVC's are RCSJ-like and exhibit hysteresis at low $H$. Inset shows a sketch of the junction.

plying a gate voltage, $V_{g}$. Details of sample fabrication and characterization can be found elsewhere [28, 29].

Fig. 7 shows a set of IVC's at $T=30 \mathrm{mK}$ for different $V_{g}$. It demonstrates that the critical current is increased at positive $V_{g}$ and strongly suppressed at small negative $V_{g}$. Note that the resistance of the junction starts to increase at substantially larger negative $V_{g} \lesssim-1 V$, at which the critical current is already strongly suppressed. Therefore the IVC's at $V_{g}>-1 V$ are reasonably well described by the RCSJ model with a constant $R$.

The majority of the switching current measurements were performed on the junction $(\# 2 b)$ with a wide $(40 \mu \mathrm{m})$ and short $(400 \mathrm{~nm})$ 2DEG and good transparency of the $\mathrm{Nb} / 2 \mathrm{DEG}$ interface. For $40 \mu \mathrm{m}$ wide junctions, shunting by high frequency impedance was insignificant because of the small junction resistance $R \simeq 7.5-10 \Omega$. Junctions with narrower 2DEG $(10 \mu m)$ had proportionally smaller $I_{c 0}$ and larger $R \sim 30-40 \Omega$. All JJ's studied here had a uniform critical current distribution, as judged from periodic Fraunhofer modulations $I_{c}(H)$, see Fig. 8 .

Fig. 9 shows $T$-dependencies of $I_{S}$ and $I_{R}$ for the same JJ at two magnetic fields, marked by circles in Fig. 8. The $T$-dependence of $I_{S}$ and $I_{R}$ for S-2DEG-S JJ's is similar to that of planar SFS JJ's, see Fig. 3. In both cases the $I_{R}$ is $T$-independent in a wide $T$ - range.

\section{Bi-2212 intrinsic Josephson junctions}

Intrinsic Josepson junctions (IJJ's) are naturally formed between adjacent $\mathrm{Cu}-\mathrm{O}$ layers in strongly anisotropic HTSC single crystals [30]. IJJ's behave as SIS-type junctions [31, 32] with high $Q_{0}$, inspite of the d-wave symmetry of the order parameter in HTSC. This
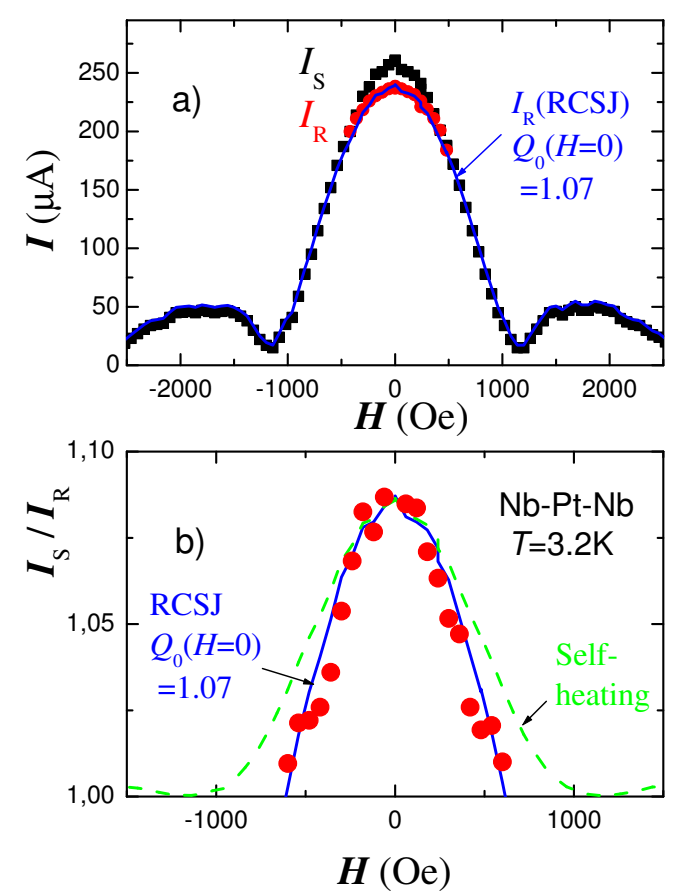

FIG. 6: (Color online). a) Magnetic field modulation of the switching and retrapping currents for the same $\mathrm{Nb}-\mathrm{Pt}-\mathrm{Nb}$ junction as in Fig. 5. b) $I_{S} / I_{R}$ vs $H$. The lines represent calculations within the RCSJ model for $Q_{0}(H=0)=1.07$ (solid), and in case when the hysteresis is solely caused by self-heating (dashed), see sec. IV. It is seen that the capacitive hysteresis within the RCSJ model disappears abruptly at certain $I_{c}(H)$, while the self-heating hysteresis decreases gradually with $I_{c}$.

was confirmed by observation of geometric Fiske resonances [33, 34] and energy level resolution in the MQT experiments on Bi-2212 IJJ 35] and $\mathrm{YBa}_{2} \mathrm{Cu}_{3} \mathrm{O}_{7-\delta}$ biepitaxial c-axis (IJJ-like) JJ's [20].

IJJ's were made by micro/nano-patterning of small mesa structure on top of Bi-2212 single crystals. Here we present data for an optimally doped Bi-2212 single crystal with $T_{c} \simeq 94.5 \mathrm{~K}$. Mesas were cut in two parts by FIB to allow true four-probe measurements. Details of mesa fabrication can be found elsewhere 36. Properties of our IJJ's were described in detail before 31, 32]. Fig. 10 shows IVC's at different $T$ for a Bi-2212 mesa. IVC's of IJJ's are non-linear and exhibit strong hysteresis below $T_{c}$. However, at elevated temperatures, $70<T<T_{c}$, the IVC's are almost linear in a small voltage range [2, 31]. Each mesa contains several stacked IJJ's. Therefore, IVC's exhibit a multi-branch structure due to one-by-one switching of stacked IJJ's from $S$ to $R$ state.

Fig. 11 shows the $T$-dependence of the most probable switching current, $I_{S \max }$, the retrapping current $I_{R}$, and the fluctuation-free critical current $I_{c 0}$ for a single IJJ from the same Bi-2212 sample as in Fig. 10 [37]. The $I_{c 0}$ was obtained from the analysis of switching statis- 


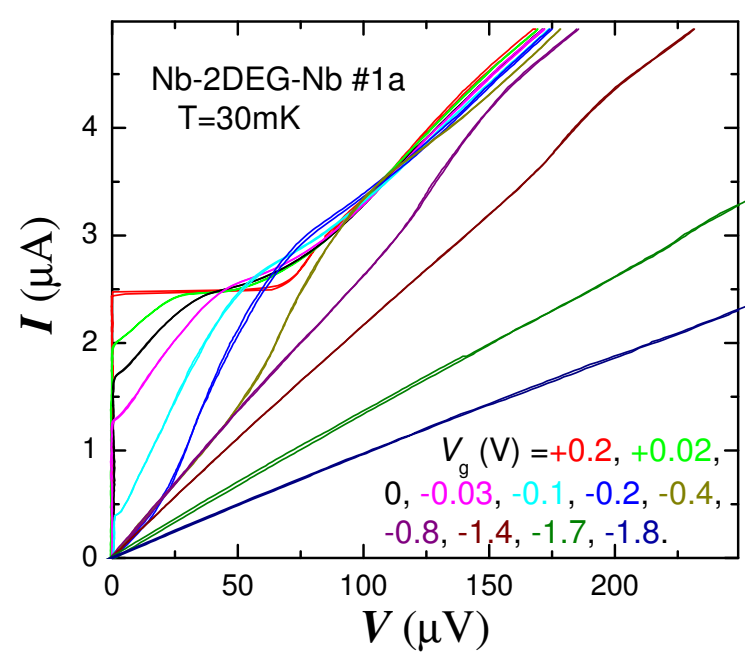

FIG. 7: (Color online). The IVC's of a S-2DEG-S junction for different gate voltages at $T=30 \mathrm{mK}$.

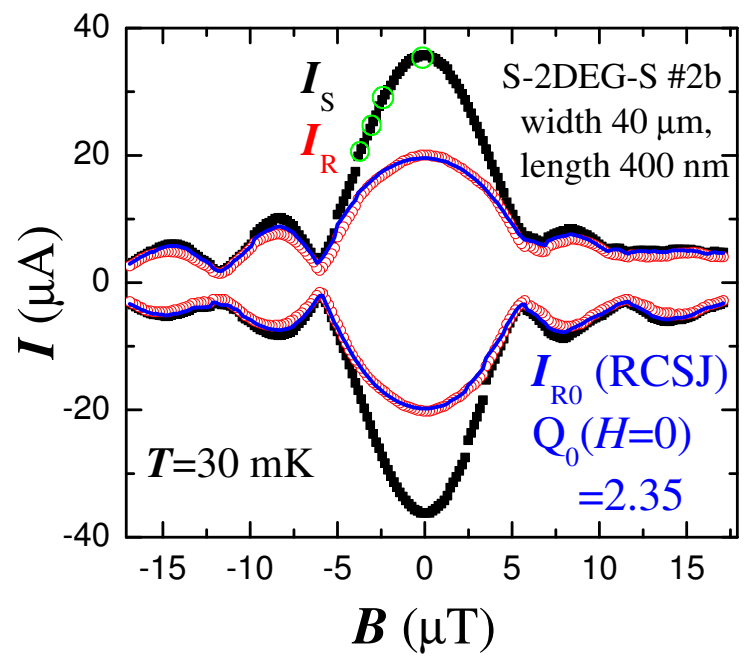

FIG. 8: (Color online). Magnetic field modulation of switching and retrapping currents for the S-2DEG-S junction \#2b at $T=30 \mathrm{mK}$. The solid line represents a simulation within the RCSJ model for $Q_{0}(H=0)=2.35$.

tics 36. The $I_{c 0}(T)$ follows the Ambeokar-Baratoff dependence typical for conventional SIS JJ's 36]. The $I_{R}$ is $T$-independent at low $T$ and increases with $T$ up to $\sim 85 \mathrm{~K}$. Such behavior is also typical for conventional SIS JJ's and is attributed to strong $T$-dependence of the low bias quasiparticle resistance, which determines the effective dissipation for the retrapping process [38].

\section{THE ORIGIN OF HYSTERESIS}

Figs. 2-11 show that the IVC's of all the four types of JJ's studied here exhibit a hysteresis at low $T$. According to the RCSJ model the hysteresis is related to damping

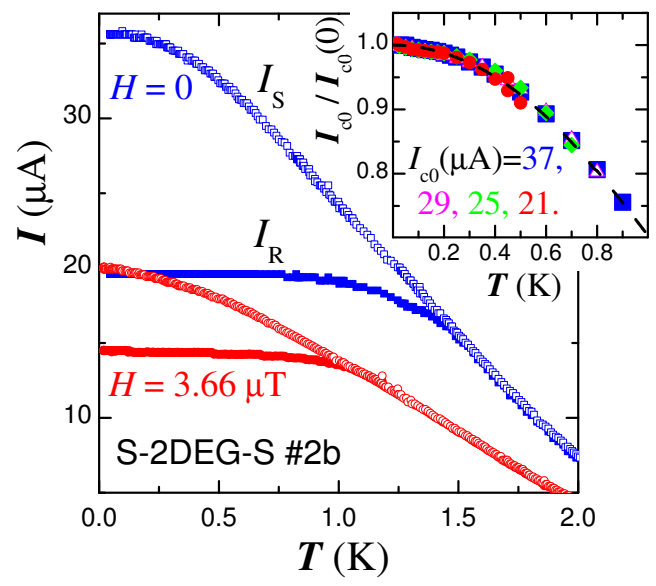

FIG. 9: (Color online). T-dependence of the switching and retrapping currents for the S-2DEG-S junction \#2b at magnetic fields marked by two of the circles in Fig. 8 . The inset shows $T$-dependencies of normalized fluctuation-free critical currents $I_{c 0}(T) / I_{c 0}(T=0)$ at four $H$ marked in Fig. 8 .

and appears in underdamped JJ's with $Q_{0}>0.84$.

For the case of Bi-2212 IJJ's the hysteresis can be unambiguously attributed to a large capacitance caused by atomic scale separation between electrodes. From measurements of Fiske step voltages [33] the specific capacitance of our IJJ's was estimated as $C \sim 68.5 \mathrm{fF} / \mu \mathrm{m}^{2}$. Substituting typical parameters of IJJ's 32], the critical current density $J_{c}(4.2 \mathrm{~K}) \simeq 10^{3} \mathrm{~A} / \mathrm{cm}^{2}$; the large bias $c$-axis tunnel resistivity $\rho_{c} \simeq 30 \Omega c m$ and the stacking periodicity $s \simeq 1.5 \mathrm{~nm}$, we obtain $Q_{0}(4.2 \mathrm{~K}) \simeq 20$. This value will become up to two orders of magnitude larger if we use the low bias quasiparticle resistivity at $T=4.2 \mathrm{~K}$ instead of $\rho_{c}$. In any case, IJJ's are strongly underdamped, $Q_{0} \gg 1$, at $T \ll T_{c}$ and also remain underdamped in practically the whole $T$-range $T<T_{c}$, as seen from Fig. 11. It has been demonstrated that the hysteresis $I_{S} / I_{R}$ in Bi-2212 IJJ's agrees well with the calculated $Q_{0}$ using the specific capacitance of IJJ's 36 .

On the other hand, explanation of the hysteresis in SNS-type JJ's is less straightforward. Typically SNS JJ's are strongly overdamped, $Q_{0} \ll 1$, and the hysteresis is caused by either self-heating 39], non-equilibrium effects [40], or frequency dependent damping [9], rather than the junctions capacitance.

\section{Self-heating}

It is known that self-heating can cause hysteresis in superconducting weak links with negligible $C$ [39]. In this case the "retrapping" current simply represents $I_{S}$ at the elevated temperature due to power dissipation at the resistive branch of the IVC: 


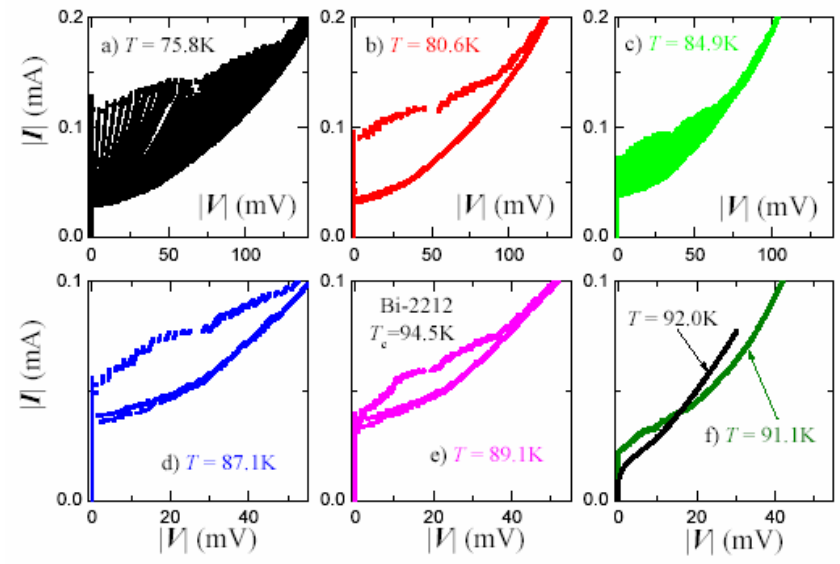

FIG. 10: (Color online). Four-probe $I-V$ characteristics of a Bi-2212 mesa at different temperatures. It is seen that the multi-branch structure and the hysteresis persists up to $\sim 3 K$ below $T_{c}$.

$$
\left.I_{R}=I_{S}\left(T_{0}+\Delta T\left(I_{R}\right)\right) \simeq I_{S}\left(T_{0}\right)+\frac{d I_{S}}{d T} \Delta T\left(I_{R}\right)\right) .
$$

The temperature rise is given by $\Delta T\left(I_{R}\right)=P_{R} R_{t h} \simeq$ $R R_{t h} I_{R}^{2}$, where $R_{t h}$ is the thermal resistance of the junction and $P_{R}$ is the power dissipation at $I_{R}$. Thus, Eq. (16) for $I_{R}$ becomes:

$$
I_{R} \simeq I_{S}\left(T_{0}\right)\left[1-\alpha I_{R}^{2}\right],
$$

where $\alpha=-\left(d I_{S} / d T\right) R R_{t h} / I_{S}\left(T_{0}\right)$. The solution of this quadratic equation yields:

$$
I_{R} \simeq \frac{\sqrt{1+4 \alpha I_{S}^{2}}-1}{2 \alpha I_{S}}
$$

The dashed lines in Figs. $4 \mathrm{~b}$ ) and $6 \mathrm{~b}$ ) show fits to experimental $I_{R}(H)$ in the self-heating model, Eq. (18). The solid lines in the same figures represent fits within the RCSJ model, Eqs. $(1,10) . I_{R}(H)$ for both self-heating and RCSJ models were obtained using a single fitting parameter $\left(R_{t h}\right.$ and $Q_{0}(H=0)$, respectively), which are unambiguously determined from hysteresis $I_{S} / I_{R}$ at $H=0$. The self-heating and RCSJ models provides almost equally good fits to the $I_{R}(H)$ modulation for the SFS junction in Fig. 4. Similarly, $T$-dependences of $I_{R}$ within the two scenarios are practically indistinguishable for this sample, as seen from comparison of dashed and solid lines in Fig. 3.

For the SNS JJ, the RCSJ model provides a better fit to the experimental data than the simple self-heating model, see Fig. 6b). In experiment and in the RCSJ model the hysteresis disappears abruptly at certain $H$, while selfheating is always present and leads to smooth variation

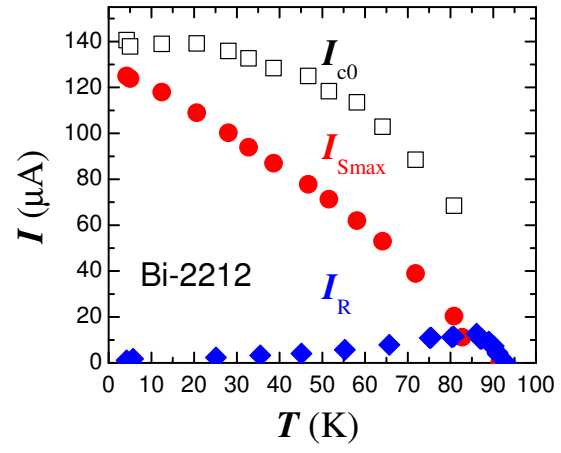

FIG. 11: (Color online). Temperature dependence of the most probable switching $I_{S \max }$, retrapping $I_{R}$ and fluctuation-free critical $I_{c 0}$ currents for the same Bi-2212 IJJ.

of the hysteresis near the minima. On the other hand, a better fit can be obtained if we allow some $T$-dependence of $R_{t h}$.

A clue to the origin of hysteresis can be obtained from comparison of IVC's of JJ's with identical geometry but different $I_{S}$. As described in the previous section, for planar SFS JJ's, a minor variation of the FIB-cut depth changes $I_{c 0}$ by several orders of magnitude without affecting other characteristics of JJ's $\left(C, R\right.$ and $\left.R_{t h}\right)$, as seen from Figs. 2 and 3. The inset in Fig. 3 shows the values $1-I_{R} / I_{S}$ at $T \simeq 30 \mathrm{mK}$ for three SFS JJ's with similar geometry on the same chip. Within the self-heating scenario, Eq.(16), $1-I_{R} / I_{S}$ would be proportional to the power at retrapping, $P_{R}$, as shown by the dashed line in the inset to Fig. 3. This seems to be true for JJ's with large $I_{c 0}$ and $P_{R}$. However, provided that the hysteresis in JJ's with larger $I_{c 0}$ is caused by self-heating and $R_{t h}$ of all JJ's are the same, there should be no hysteresis due to self-heating for the JJ with the smallest $I_{c 0}$ in Fig. 3. This is indicated by the green (lower) dashed line in the main panel of Fig.3, which represents the self-heating $I_{R}(T)$ for the JJ with small $I_{S}$, calculated from Eq.(18) using the parameter $\alpha$ obtained from the fit $I_{R}(T)$ for the JJ with larger $I_{S}$, shown by the black (upper) dashed line in Fig. 3.

Furthermore, experimental $I_{R}(T)$ for our junctions are almost $T$-independent at low $T$. This is in contrast to the prediction of the self-heating model, Eq.(18): $I_{R} \propto$ $I_{S}^{1 / 2}$, see the black (upper) dashed line in Fig. 3. Note that the flatter $I_{R}(T)$ dependence can not be explained by the flatter $I_{c 0}(T)$ in comparison to $I_{S}(T)$, because within the self-heating model $I_{R}$ is correlated with $I_{S}$, not $I_{c 0}$. Exactly the same behavior was observed for S2DEG-S junctions, see Fig. 9.

Thus, we conclude that self-heating does not satisfactory explain the hysteresis in SFS junctions, although it is probably responsible for a considerable part of the hysteresis in JJ's with larger $I_{s}$.

For Bi-2212 IJJ's, self-heating was measured directly 
[41] for the same mesa. The $R_{t h}$ of the mesa ranged fro] $\sim 70 \mathrm{~K} / \mathrm{mW}$ at $\mathrm{T}=4.2 \mathrm{~K}$ to $\sim 10 \mathrm{~K} / \mathrm{mW}$ at $80 \mathrm{~K}$. Sinc the dissipated power at $I<I_{S}$ at the first branch $\mathrm{j}$ the IVC never exceeded a few $\mu W$, self-heating can $k$ excluded as the origin of hysteresis in IJJ's.

\section{Capacitance}

If the hysteresis were due to finite junction capacitanc then magnetic field modulation of $I_{R 0}(H)$ should be unique function of $I_{c 0}(H)$, given by Eqs. $(9,10)$ with fie] dependent $Q_{0}(H)=Q_{0}(H=0)\left[I_{c 0}(H) / I_{c 0}(H=0)\right]^{1 /}$ as follows from Eq.(1). The corresponding $I_{R 0}(H)$ curv calculated within the RCSJ model are shown by solj lines in Figs. 4, 6, and 8. In all cases the agreeme1 with the experimental data is remarkable, considerir that there is only one fitting parameter $Q_{0}(H=0) \mathrm{fc}$ each curve (indicated in the figures).

Next we estimate the capacitances that would be $\mathrm{r}$ quired for reaching $Q_{0}=1: C\left[Q_{0}(H=0)=1\right] \sim 35 p$. for SFS \#2a (Fig.4), $\sim 4 p F$ for SNS (Fig.6) and $\sim 0.2 p r$ for S-2DEG-S \#2b (Fig.8) JJ's, respectively. Those must be compared with the expected geometrical $C$ of the JJ's.

The overlap capacitance of the SNS junction, Fig.6, is small, $\sim$ a few $f F$, due to small area of the JJ $(\sim$ $\left.0.015 \mu \mathrm{m}^{2}\right)$. The stray capacitance was estimated to be of the same order of magnitude. Therefore, the total $C$ of this junction is insufficient for observation of the hysteresis within the simple RCSJ model.

The total (stray) capacitance of wide S-2DEG-S JJ's with the gate electrode is estimated to be $\sim 0.1-0.2 p F$. This $C$ can cause a substantial hysteresis in the junction \#2b with large $I_{c 0}$ and may be just sufficient for a tiny hysteresis in the other junctions with smaller $I_{c 0}$. This conclusion is also supported by observation of underdamped phase dynamics in those junctions, as will be discussed below.

We argued above that the hysteresis in SFS JJ's can not be caused solely by self-heating. But how could the huge $C \sim 35 p F$ appear in those planar JJ's with the stray capacitance in the range of few $f F$ ? To understand this we should consider the specific junction geometry, shown in Fig. 2 a). The JJ's are made of $\mathrm{Nb} / \mathrm{CuNi}$ bilayers. The CuNi-layer may act as a ground plane for the JJ and may create the large overlap capacitance, provided there is a certain barrier for electron transport between the layers. The transparency of $\mathrm{Nb} / \mathrm{Cu}$ interfaces, made in the same setup, was previously estimated to be $\sim 0.4$ [42, 43, 44]. The interface transparency between $\mathrm{Nb}$ and $\mathrm{CuNi}$ is expected to be even smaller due to appearance of excess interface resistance between normal metals and spin-polarized ferromagnets [45]. For typical values of the overlap capacitance $C \sim 20-40 \mathrm{fF} / \mu \mathrm{m}^{2}$, the required $C \sim 35 p F$ can originate from the bilayer within just $\sim 30-40 \mu m$ radius from the JJ. An unambiguous

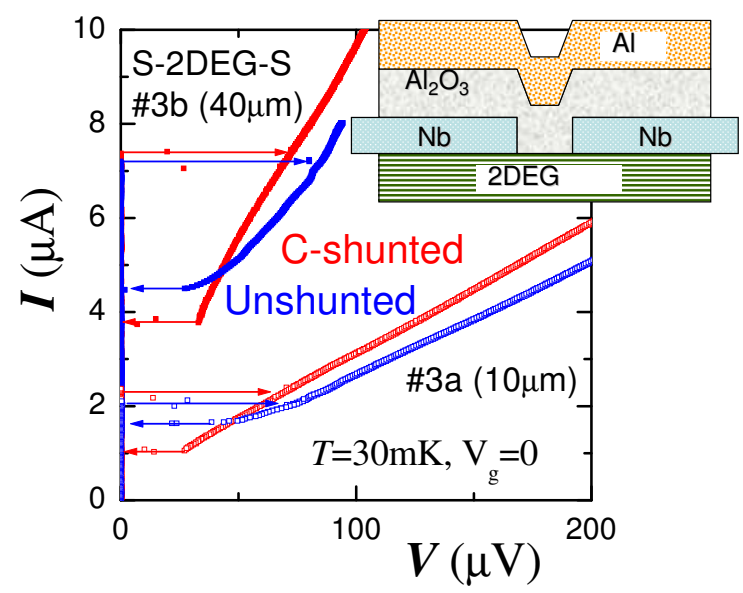

FIG. 12: IVC's of two S-2DEG-S junctions on the same chip at $T=30 \mathrm{mK}, H=0$, before and after in-situ $C$ - shunting. Inset shows a sketch of the $C$-shunted junction.

confirmation of the presence of the large $C$ in our SFS JJ's follows also from observation of the underdamped phase dynamics, as reported below (Fig. 16).

\section{In-situ capacitive shunting}

The frustratingly similar behavior of $I_{R}(T, H)$ within self-heating and RCSJ models hinders discrimination between self-heating and capacitive origins of the hysteresis. To clarify the origin of hysteresis, we fabricated an in-situ shunt capacitor, consisting of $300 \mu \mathrm{m}$ wide $\mathrm{Al}_{2} \mathrm{O}_{3} / \mathrm{Al}$ double layer deposited right on top of S-2DEG-S JJ's. The sketch of the $C$-shunted junction is shown in Fig. 12 . The IVC's for two JJ's from the same chip before and after $C$ - shunting are shown in Fig. 12. The length of 2DEG in those junctions was $500 \mathrm{~nm}$, which results in considerably smaller $I_{c 0}$ than for the JJ\#2b with $400 \mathrm{~nm}$ long 2DEG, Fig.8. It is seen that the hysteresis for both junctions increased considerably, while $R$ was little affected by $C$ - shunting [46]. The total capacitance of the shunt was $\sim 5 p F$, much larger than the initial capacitance of unshunted junctions $C \sim 0.1-0.2 p F$.

It should be emphasized that introduction of the $C$-shunt improve thermal conductance from the junctions. Indeed, since the sample was placed in vacuum, the $C$-shunt double layer on top of the $2 \mathrm{DEG}$ acts as the top heat spreading layer [47, 48], creating an additional heat sinking channel. Thus, the self-heating hysteresis must decrease in the $C$-shunted JJ. Therefore, increase of the hysteresis in $C$-shunted JJ's unambiguously indicates that the hysteresis is indeed caused by the junction capacitance, rather than self-heating.

To summarize this section, the hysteresis in the IVC's of IJJ's are caused solely by the junction capacitance. The $C$-shunted S-2DEG-S JJ's are underdamped with 
predominantly capacitive hysteresis. For most SNS-type junctions the hysteresis is considerably affected by selfheating. However, planar SFS and S-2DEG-S junctions with short $(400 \mathrm{~nm})$ 2DEG are underdamped, $Q_{0} \gtrsim 1$, at low $T$. Therefore, a substantial part of hysteresis in those junctions has a capacitive origin. This conclusion is confirmed below by observation of the underdamped phase dynamics in those junctions.

\section{COLLAPSE OF THERMAL ACTIVATION}

Measurements of switching and retrapping current statistics were made in a carefully shielded dilution refrigerator (sample in vacuum) in a shielded room environment. Measurements in the temperature range 1.2-100 K were measured in a $\mathrm{He}^{4}$ cryostat (sample in liquid or gas). Switching and retrapping currents were measured using a standard sample-and-hold technique. All histograms were made for 10240 switching events.

\section{Collapse in Bi-2212 intrinsic Josephson junctions}

A sporadic switching of simultaneously biased stacked IJJ's in the Bi-2212 mesa results in rather chaotic switching between quasiparticle branches in the IVC, see Fig.10. This makes the analysis of switching statistics quite complicated. In addition, strong electromagnetic coupling of atomic scale stacked IJJ's in the mesa leads to appearance of metastable fluxon states $[49,52$ and results in multiple valued critical current [49, 51]. It has been reported that switching histograms of IJJ's can be very broad and contain multiple maxima [49, 50, 51], consistent with frustration caused by the presence of metastable states in long, strongly coupled stacked JJ's [49, 51].

In order to avoid the metastable states, we studied switching statistics of a single IJJ [36, 37]. In the studied mesa, one of the junctions occasionally had slightly smaller $I_{c 0}$ (by $\sim 20 \%$ ) than the rest of the IJJ's. Thus, we were able to achieve stable switching of this single IJJ, while the rest of the IJJ's remained in the $S$-state [36].

Fig. 13 shows switching histograms for the single IJJ at different $T$. The black solid lines represent simulated histograms for conventional TA escape, Eqs.(213), at given $T$, and for corresponding junction parameters and experimental sweeping rates. Detailed analysis of the switching histograms can be found elsewhere [36]. The switching histograms are perfectly described by the TA theory up to $T^{*} \sim 75 \mathrm{~K}$. However, at higher $T$ the histograms suddenly become narrower. This is clearly seen from Fig. $13 \mathrm{~b}$ ) which represents the effective escape temperature $T_{e s c}$ obtained from the fit of experimental escape rate by the TA expression Eq.(2), with $T=T_{\text {esc }}$ being the fitting parameter. For conventional TA, $T_{e s c}=T$, as

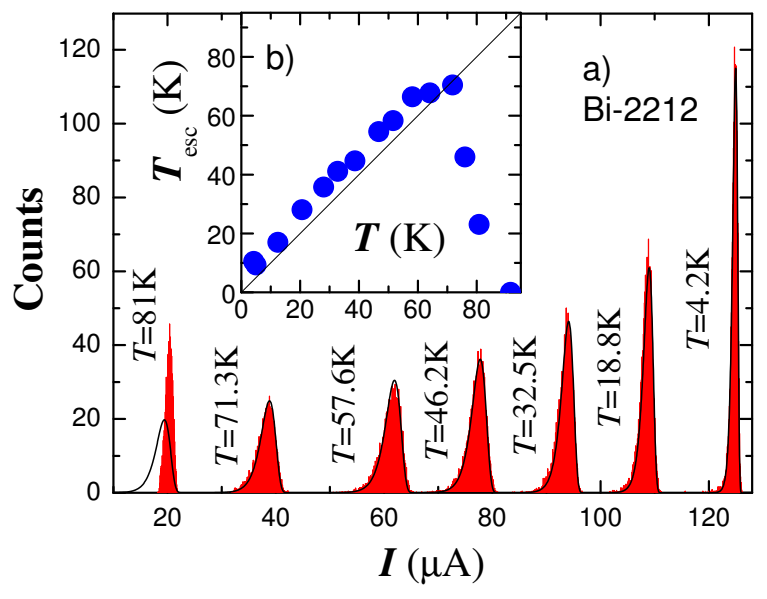

FIG. 13: (Color online). a) Switching histograms of a single IJJ at different $T$. The solid lines represent theoretical results for thermal activation at a given $T$. Note that the experimental histograms initially become wider with increasing $T$, but suddenly become narrower and change the shape at $T^{*}$ between $71 K$ and $81 K$. b) The effective escape temperature vs. $T$ extracted from fitting the switching histograms to the TA theory. The solid line represents the prediction of conventional TA theory, $T_{e s c}=T$. A sudden collapse of $T_{e s c}$ is seen at $T^{*} \simeq 75 \mathrm{~K}$.

shown by the solid line in Fig. $13 \mathrm{~b}$ ). A sudden collapse of $T_{\text {esc }}$ at $T^{*} \simeq 75 \mathrm{~K}$ is clearly seen from Fig. $13 \mathrm{~b}$ ). We also note that the histograms become progressively more symmetric at $T>T^{*}$.

\section{Collapse in S-2DEG-S junctions}

S-2DEG-S junctions provide a unique opportunity to tune $I_{c 0}, E_{J 0}$ and $Q_{0}$ by applying the gate voltage $V_{g}$, see Fig. 7. Fig. 14 a) shows switching current histograms at $T=37 m K$ for the S-2DEG-S \#3b'(identical to \#3b) at different $V_{g}$. Panel b) shows that the most probable switching current, $I_{S \max }$, decreases monotonously with increasing negative $V_{g}$. Panel c) shows the width at halfheight, $\Delta I$, versus $I_{S \max }$. It is seen that initially histograms are getting wider with increasing negative $V_{g}$, due to the increase of TA with decreasing $E_{J 0} / T$. However, at $V_{g}<-0.35 \mathrm{~V}$ a sudden change occurs and $\Delta I$ starts to rapidly collapse.

Fig. 15 a) shows $T_{e s c}$ vs. $T$ for the S-2DEG-S \#2b at $H=0,2.32,3.05$, and $3.66 \mu T$ (marked in Fig.8). In all cases we can distinguish three $T$ - regions:

(i) The MQT regime. At low $T, T_{e s c}$ is independent of $T$. Both the TA-MQT crossover temperature, at which saturation of $T_{\text {esc }}(T)$ occurs with decreasing $T$, and the value of $T_{\text {esc }}(T \rightarrow 0)$ decrease with $H$, which leaves no doubts that we observe the MQT state [7, 9, 10, 11]. Fig. $15 \mathrm{~b}$ ) shows the dependence of the $T_{e s c}$ at $T=20 \mathrm{mK}$ as a function of $I_{c 0}(H) . I_{c 0}$ for each $H$ was extrapolated from 

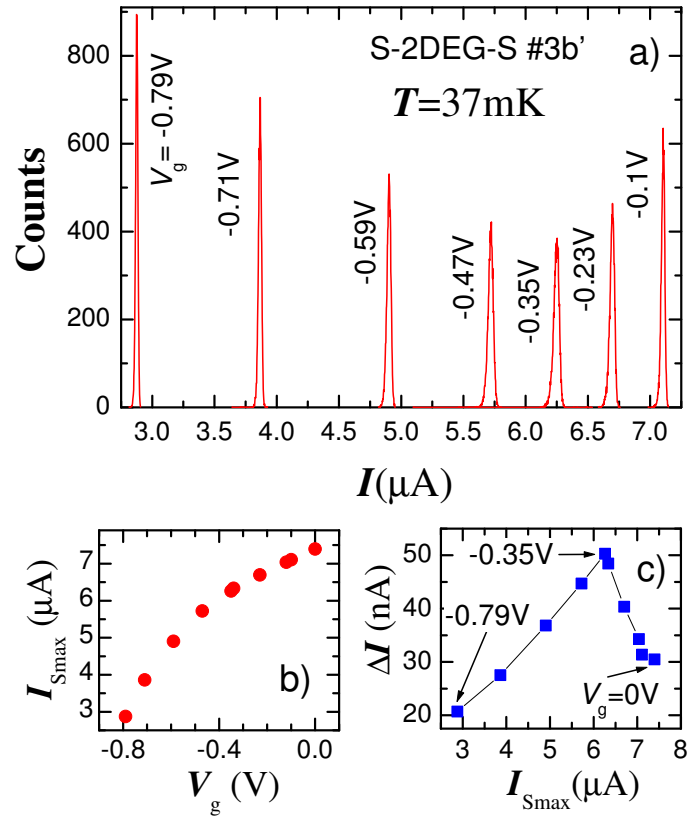

FIG. 14: (Color online). a) Switching histograms of S-2DEG$\mathrm{S} \# 3 \mathrm{~b}$ ' at $T=37 \mathrm{mK}$ for different gate voltages $V_{g}$. It is seen that the height (inversely proportional to the width) of the histograms first decreases, but then start to increase with increasing negative $V_{g}$. b) Dependence of the most probable switching current $I_{S \max }$ on the gate voltage. c) The width of the histograms $\Delta I$ vs. $I_{S \max }$. A sudden collapse of $\Delta I$ occurs at $V_{g}<-0.35 \mathrm{~V}$.

the switching histograms. The inset in Fig. 9 demonstrates that $I_{c 0}(T) / I_{c 0}(T \rightarrow 0)$ for different $H$ collapse into one curve, confirming the accuracy of determination of $I_{c 0}(T, H)$. The solid line in Fig. $15 \mathrm{~b}$ ) shows the fit to the TA-MQT crossover temperature, Eq.(6), taking $Q_{0}(H=0)=2.35$, following from the value of hysteresis in the IVC's, see Fig. 8. Obviously, the MQT calculations are consistent with the previous conclusion that this JJ is underdamped at low $T$ and that the hysteresis is predominantly caused by the junction capacitance.

We tried to perform quantum level spectroscopy [20] in the MQT state for this JJ, but couldn't observe any interlevel transitions. This implies that the level width $\sim \hbar /(R C)$ is of the order of level spacing $\hbar \omega_{p}$, which in turn is caused by a relatively low $Q_{0}$ for this JJ. Therefore, Fig. 15 confirms the theoretical prediction [7, 8] that the MQT occurs even in the absence of well defined quantum levels in slightly underdamped and overdamped JJ's, $Q_{0} \lesssim 1$. Both the absolute value and the characteristic parabolic shape of $T_{e s c}(T)$ in the MQT state are in good agreement with theoretical predictions, as shown in Fig. 15 c). The solid lines in Fig.15 c) show simulated $T_{\text {esc }}$ vs $T$ in the MQT state, calculated from Eq.(5) using the experimental conditions for the data in Fig. 15 a). It is seen that both the absolute value and the shape of simulated $T_{e s c}(T)$ agree with the experimental data.
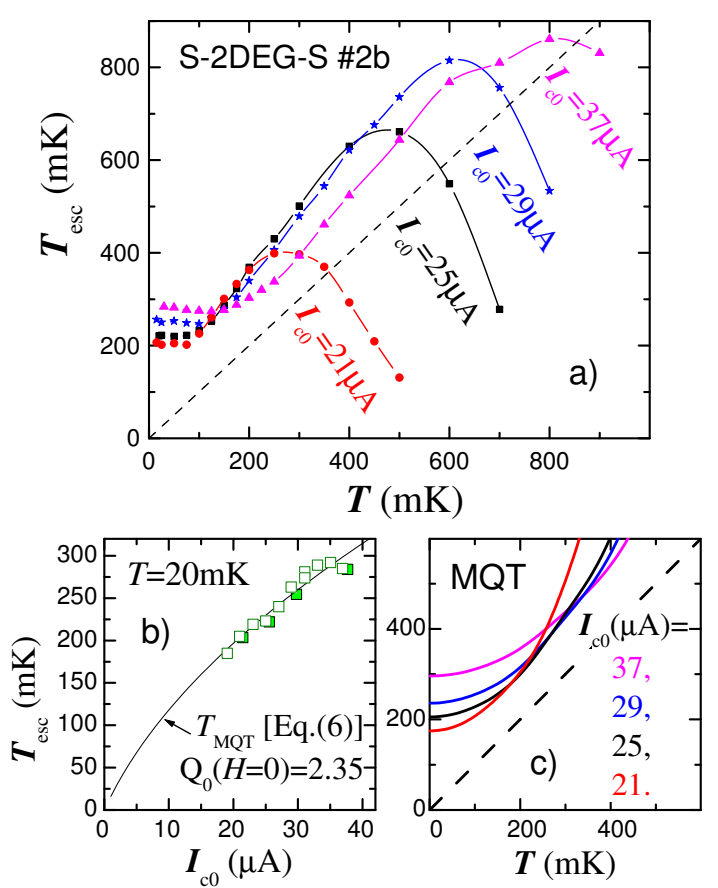

FIG. 15: (Color online). a) Escape temperature vs. $T$ for the S-2DEG-S JJ \#2b at four magnetic fields (marked by circles in Fig. 8). Three $T$ - regions can be distinguished: the MQT at low $T$, the TA at intermediate $T$, and the collapse region at $T>T^{*}$. b) $T_{\text {esc }}$ in the MQT state at $T=20 \mathrm{mK}$ as a function of the fluctuation free $I_{c 0}$, suppressed by applying magnetic field. Solid line represents the fit to Eq.(6) for $Q_{0}(H=0)=2.35$. c) Results of MQT simulations, Eq.(5), for the experimental conditions in panel a).

(ii) Thermal activation regime. At intermediate $T$, $\Delta I$ increases in agreement with TA calculations, $T_{e s c}=$ $T$ shown by dashed lines in Fig. 15 a). Slightly larger inclination of the experimental $T_{e s c}(T)$ may be due to non-sinusoidal CPR in this SNS-type JJ [22].

(iii) Collapse of thermal activation. At higher $T$, the width of histograms start to rapidly collapse, leading to a downturn of $T_{e s c}(T)$. The magnetic field dependence from Fig. 15 a) reveals that the collapse temperature $T^{*}$ decreases quite rapidly with $I_{c 0}$, i.e., the collapse occurs at lower $T$ in junctions with smaller $Q_{0}$.

\section{Collapse in planar SFS junctions}

Fig. 16 shows switching current statistics at different $T$ for the planar Nb-CuNi-Nb junction \#2a at $H \simeq 0.5 O e$. The scales of both axes were kept constant for all histograms to facilitate direct comparison of the histograms at different $T$. It is seen that the collapse of TA occurs at $T^{*} \simeq 200 m K$. 

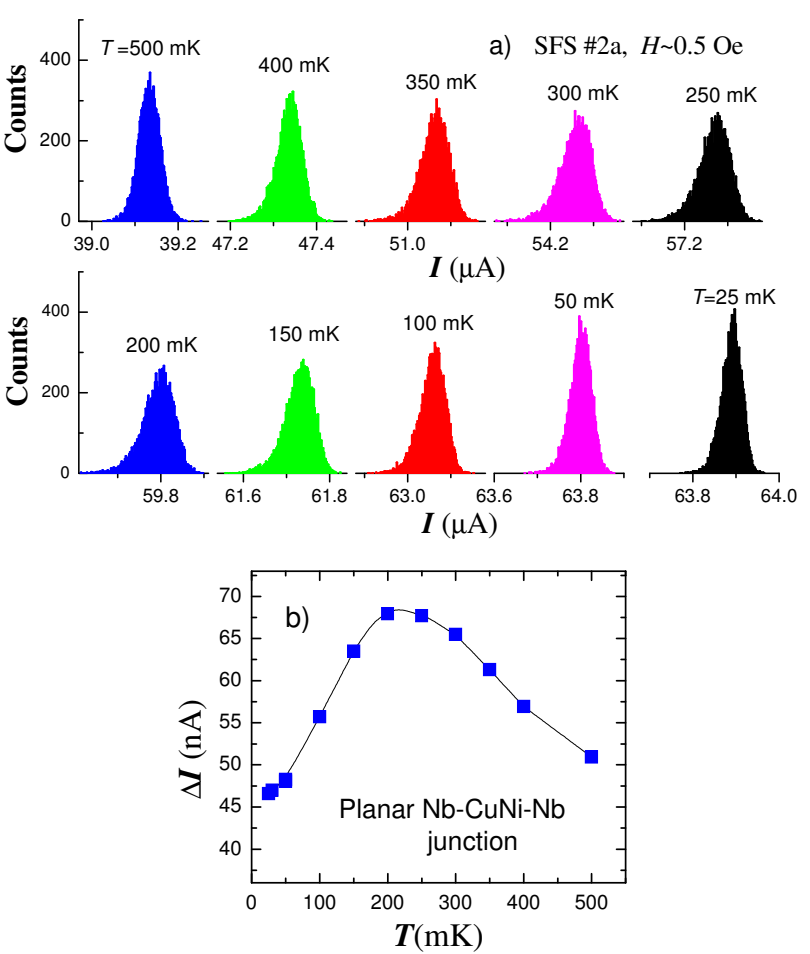

FIG. 16: (Color online). a) Switching histograms of the planar SFS junction \#2a at different $T$. The histograms become wider with increasing $T$ up to $T=200 \mathrm{mK}$ (lower row) but then start to shrink at $T>250 \mathrm{mK}$ (upper row). b) The width of histograms $\Delta I$ vs. $T$. The $\Delta I(T)$ follows the TA behavior up to $T^{*}=200 \mathrm{mK}$ but collapses at higher $T$.

\section{The shape of switching histograms}

Fig. 17 shows switching histograms of the same single IJJ as in Fig.13 just before and after the collapse. It demonstrates that not only the width, but also the shape of the histogram changes upon the collapse. At $T<T^{*}$ the histograms have the characteristic asymmetric shape, perfectly consistent with the TA theory [36], as shown by the black dashed line (coincides with the blue solid line) in Fig. 17 a). However, at $T>T^{*}$ histograms become narrower and loose the characteristic asymmetric shape, as seen from Fig. $17 \mathrm{~b}$ ). Such a tendency was observed for all JJ's, as can be seen from Figs. 13,14,16. As will be discussed in sec. VI below, the transformation of the histogram shape at $T>T^{*}$ is caused by the interference of switching and retrapping processes.

\section{Effect of $C$-shunting}

Fig.18 shows $T_{\text {esc }}$ vs $T$ obtained from switching current statistics, for the same S-2DEG-S JJ's as in Fig.12, before and after capacitive shunting. Apparently, $C$-shunting qualitatively changed the phase dynamics of the junctions, even though it had a minor effect on $I_{c 0}$ and $R$.

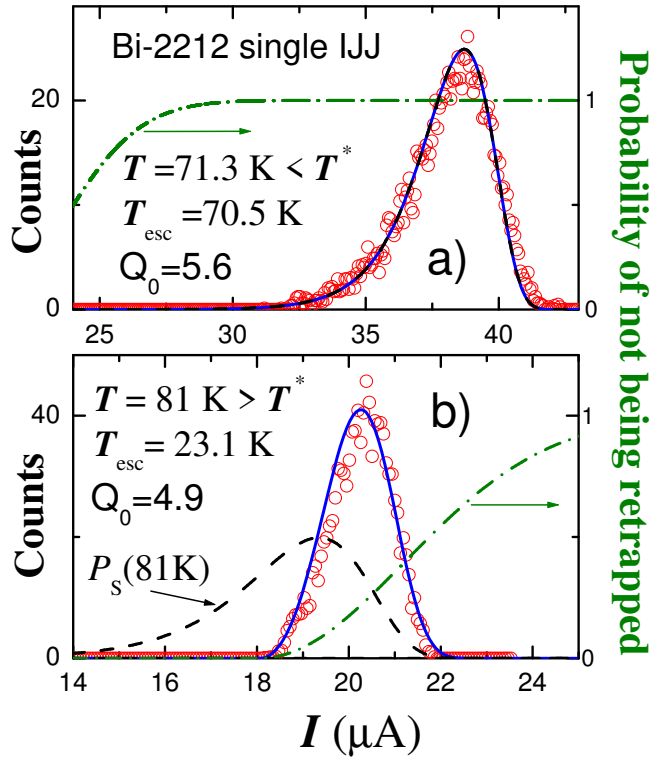

FIG. 17: (Color online). Switching histograms of a single IJJ a) below and b) above the collapse temperature $T^{*} \simeq 75 \mathrm{~K}$. Symbols represent experimental data, dashed lines - TA probability density of $S \rightarrow R$ switching, and dashed-dotted lines probabilities of not being retrapped $\mathbb{P}_{n R}$, Eq. (15). Solid lines show the conditional probability density $P_{S R}$ of switching without being retrapped. It is seen that close to the collapse temperature, the retrapping process becomes significant and effectively "cuts-off" thermal activation at small bias. Note that both the width and the shape of the histograms change at $T^{*}$. Data from Ref. [2].

However, $C$-shunting strongly affected the quality factor of the JJ's. From Fig. 18 it is seen that the switching statistics of the underdamped $C$-shunted JJ \#3b is well described by the TA theory, for which $T_{e s c}=T$. To the contrary, for the unshunted junction, which is just at the edge of being overdamped, $Q_{0} \simeq 1$, the $T_{e s c}$ decreases with increasing $T$ almost in the whole $T$-range.

\section{Failure of the thermal activation theory in moderately damped junctions}

The observed collapse can not be caused by frequency dependent damping due to shunting by circuitry impedance [9]. Indeed, we observed the collapse in planar SFS junctions with $R<1 \Omega$, for which such shunting plays no role. Neither can it be due to $T$ - dependence of the TA prefactor $a_{t}$ in Eq.(4). Indeed, damping changes only gradually through $T^{*}$ and enters only into the (logarithmic) prefactor $a_{t}$ of the TA escape rate, Eq.(2). Gradual variations of $a_{t}(T)$ do not cause any dramatic variation of the TA escape rate. Moreover, in all calculations presented here we did take into account the $Q(T)$ dependence of the TA prefactor $a_{t}$, so that $T_{e s c}$ must by definition be equivalent to $T$ for the conventional 


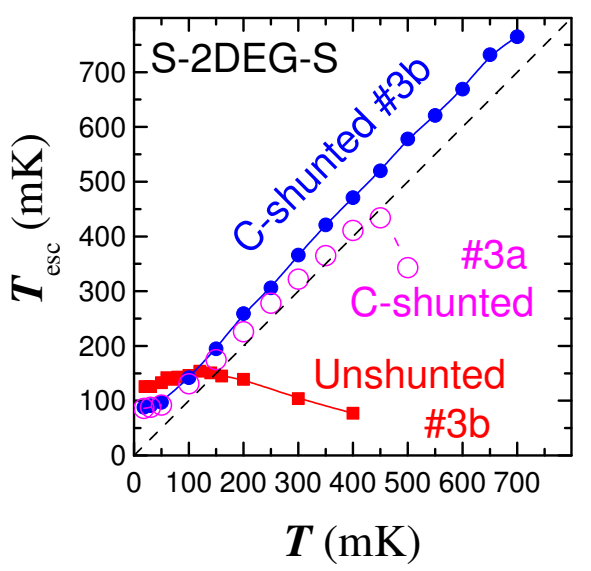

FIG. 18: (Color online). Escape temperature vs $T$ for the S-2DEG-S junctions on chip \#3 before and after in-situ $C$-shunting. It is seen that the conventional TA behavior, $T_{e s c}=T$, is restored upon increasing $Q_{0}$ after $C$-shunting.

TA and the drastic drop in $T_{\text {esc }}$ at $T>T^{*}$, can not be explained within a simple TA scenario. Therefore, the observed collapse of switching current fluctuations with increasing $T$ represents a dramatic failure of the classical TA theory, which was supposed to be valid even for overdamped JJ's [4, 7].

\section{DISCUSSION}

From Figs. 13-16 it is clear that those very different JJ's exhibit the same paradoxical collapse of switching current fluctuations with increasing $T$. A very similar collapse was observed also in moderately damped SIS type $\mathrm{Al}-\mathrm{AlO}_{x}-\mathrm{Al}[1]$ and $\mathrm{Nb}-\mathrm{AlO}_{x}-\mathrm{Nb}[3]$ junctions and SQUID's. Therefore, the collapse of TA must be a general property of all moderately damped JJ's.

The dramatic effect of $C$-shunting on the collapse $T^{*}$ clearly shows that damping has a crucial significance for the observed phenomenon. From the experimental data presented above it is also clear that $T^{*}$ decreases with increasing damping and that for overdamped junctions $T^{*} \rightarrow 0$, see the curve for unshunted JJ \#3b in Fig. 18 . The data also shows that the $T^{*}$ is close to the temperature at which the hysteresis in IVC's vanishes, compare Figs. 3 and 16, 9 and 15 a), 11 and 13, which implies that retrapping processes may become important in the vicinity of the collapse state.

\section{Influence of retrapping on the switching statistics of moderately damped junctions}

The paradoxical collapse of thermal fluctuations and the corresponding failure of the conventional TA theory in moderately damped JJ's can be explained by the in- fluence of retrapping processes on the switching current statistics [1, 2, 3]. Indeed, in moderately damped junctions $I_{c 0}$ and $I_{R 0}$ are close to each other. As discussed in sec. II, increasing $T$ tends to decrease $I_{S}$ and increase $I_{R}$. Therefore, at sufficiently high $T$, both switching and retrapping events may become possible at the same bias. If so, the criterion for measuring the switching event has to be reformulated:

The probability of switching from the $S$ to the $R$ state is a conditional probability of switching and not being retrapped back, during the time of experiment.

$$
P_{S R}(I)=P_{S}(I) \mathbb{P}_{n R}(I) .
$$

Here $P_{S R}$ is the probability density of measuring the switching event, $P_{S}$ is the probability density of switching, Eq. (13), and $\mathbb{P}_{n R}$ is the probability of not being retrapped Eq.(15).

Dashed-dotted lines in Fig. 17 a,b) show $\mathbb{P}_{n R}$, calculated for experimental parameters typical for Bi-2212 IJJ's. The corresponding quality factors are indicated in the figures. From Fig. 17 a) it is seen that at $T<T^{*}$ the $\mathbb{P}_{n R}=1$ in the region where $P_{S}>0$, therefore retrapping is insignificant. However, at $T>T^{*}$, retrapping becomes significant at small currents. The resulting conditional probability density of measuring the switching current, $P_{S R}$, Eq.(19), normalized by the total number of switching events, is shown by the solid line in Fig. 17 b). This explains very well both the reduced width and the almost symmetric shape of the measured histogram.

\section{The collapse temperature}

Fig. 19 a) shows the bias dependence of switching $\Delta U_{S}$ and retrapping $\Delta U_{R}$ barriers. As was noted in Ref. [9], there is always a current $I_{R 0}<I_{e}<I_{c 0}$ at which $\Delta U_{S}\left(I_{e}\right)=\Delta U_{R}\left(I_{e}\right)$, so that switching and retrapping process become equally probable. However, this will have an influence on the switching current statistics only in the case when the probability density of switching at this current is considerable. For the case $Q_{0}=3$, shown in Fig. 19 a), $I_{e} / I_{c 0} \simeq 0.7$. However, at low $T$ the major part of switching events will occur at $I_{S \max } \simeq I_{c 0}>I_{e}$. Such situation is seen in Fig. 17 a) for $T<T^{*}$ : the probability of not being retrapped $\mathbb{P}_{n R} \simeq 1$ at the most probable switching current $I_{S \max }$ and according to the criterium, Eq.(19), retrapping has no influence on the switching statistics.

Figs. $19 \mathrm{~b}$ and c) represent numerical simulations in which we intentionally disregarded $T$ - dependencies of $I_{c 0}=20 \mu A, Q_{0}$ and $E_{J 0}$ for simplicity of analysis. Parameters were chosen similar to that for the S-2DEG$\mathrm{S} \# 2 b$ at $H=3.66 \mu \mathrm{T}$, see Fig. 9. The two considered cases correspond to the estimated capacitance of 

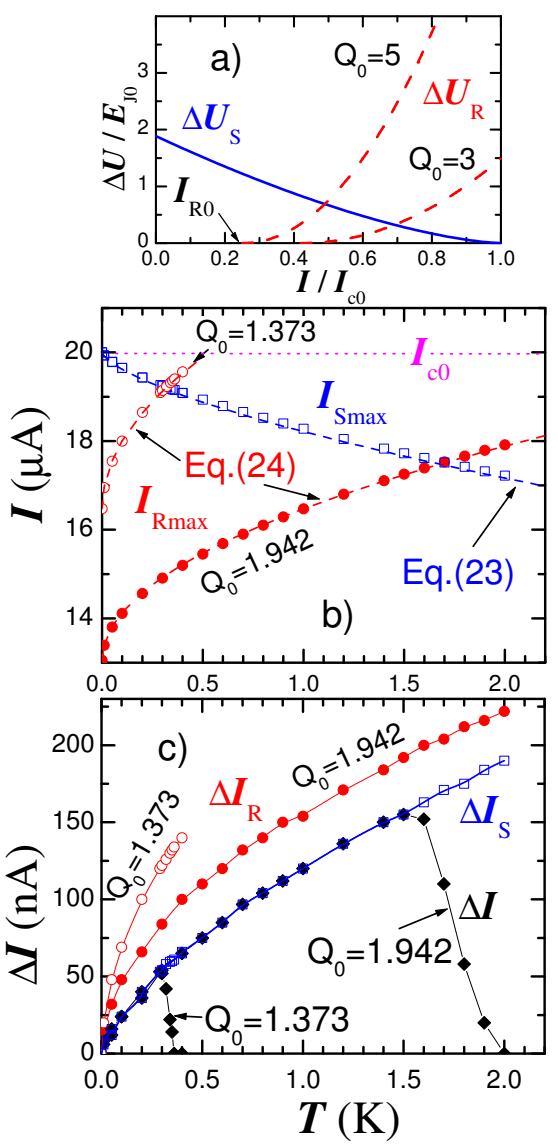

FIG. 19: (Color online). a) Escape and retrapping barrier heights as a function of bias current for $Q_{0}=3$ and 5 . b) Numerical simulations of the $T$-dependencies of the most probable switching $I_{S \max }$ and retrapping $I_{R \max }$ currents for two values of $Q_{0}$. Simulations were made for $T$-independent $I_{c 0}=20 \mu A$ (dotted line) and parameters typical for the S2DEG-S \#2b junction. c) The simulated width of switching $\Delta I_{S}$ and retrapping $\Delta I_{R}$ histograms disregarding the mutual influence of switching and retrapping processes. $\Delta I$ is the resulting width of histograms taking into account switching and retrapping. From Figs. b) and c) it is seen that the collapse of $\Delta I$ occurs at the condition $I_{S \max } \simeq I_{R \max }$.

the junction \#2b $\left(Q_{0}=1.373\right)$ and twice the capacitance $\left(Q_{0}=1.942\right)$, respectively. From Fig. 19 b) it is seen that the most probable switching current $I_{S \max }$ decreases, while the most probable retrapping current $I_{R \max }$ increases with $T$ as a result of thermal fluctuations. The width of both switching $\Delta I_{S}$ and retrappling $\Delta I_{R}$ histograms continuously increase with $T$ for conventional TA, as seen from Fig. 19 c). As expected, switching histograms are unaffected by the small variation of $Q_{0}$, so that both $I_{S \max }$ and $\Delta I_{S}$ coincide for the two values of $Q_{0}$. To the contrary, retrapping histograms are strongly affected by $Q_{0}$ : the $I_{R \max }(T)$ dependence becomes weaker and $\Delta I_{R}$ smaller with increasing $Q_{0}$. This is caused by the increase of the retrappping barrier, $\Delta U_{R}$ with $Q_{0}$ as seen from Fig. 19 a).
Since $I_{S \max }$ decreases, while $I_{R \max }$ increases with $T$, switching and retrapping histograms inevitably will overlap at a certain temperature $T^{*}$. Fig. $19 \mathrm{~b}$ and c) clearly demonstrates that the collapse of thermal fluctuations of the measured switching current $\Delta I$ occurs at $T^{*}$ and that the $T^{*}$ itself strongly depends on $Q_{0}$. From the simulations presented in Fig. 19 it is clear that the collapse is not caused by a crossover from underdamped to overdamped state since $Q_{0}$ was $T$ - independent in this case.

The collapse temperature can be estimated from the system of equations:

$$
\begin{array}{r}
\Gamma_{T A}\left(I_{\text {Smax }}\right) \simeq(d I / d t) / I_{c 0}, \\
\Gamma_{R}\left(T^{*}, I_{\text {Smax }}\right)=\Gamma_{T A}\left(T^{*}, I_{\text {Smax }}\right) .
\end{array}
$$

Eq. (20) states that the JJ switches into the R-state during the time of the experiment. From Eqs. (220) it follows that:

$$
\frac{\Delta U_{S}\left(I_{S \max }\right)}{k_{B} T} \simeq \ln \left[\frac{a_{t} \omega_{p} I_{c 0}}{2 \pi(d I / d t)}\right] \equiv Y .
$$

In the measurements presented here $Y \simeq 24$, as seen from Fig. 20 a). The parameter $Y$ is weakly (logarithmically) dependent on experimental parameters and, therefore, has approximately the same value in different studies of switching statistics of JJ's. From Eqs.(322) we obtain the value of the most probable switching current $I_{\text {Smax }}$ (disregarding retrapping):

$$
I_{S \max } / I_{c 0} \simeq 1-\left[T / T_{J}\right]^{2 / 3},
$$

where $T_{J}=\left(4 \sqrt{2} E_{J 0}\right) /\left(3 Y k_{B}\right)$. This dependence is shown by the dashed line in Fig. 19 b) and agrees with numerical simulations (squares).

Similarly, the most probable retrapping current $I_{R \max }$ (disregarding switching) is obtained from Eqs.(72122):

$$
I_{R \max } \simeq I_{R 0}+I_{c 0} \sqrt{\frac{2 k_{B} T(Y+X)}{E_{J 0} Q_{0}^{2}}},
$$

where $X=\ln \left[\frac{2 \pi\left(I_{S \max }-I_{R 0}\right)}{a_{t} I_{c 0}\left(1-\left(I_{S \max } / I_{c 0}\right)^{2}\right)^{1 / 4}} \sqrt{\frac{E_{J 0}}{2 \pi k_{B} T}}\right]$ is the $\log$ arithm of the ratio of prefactors of TA rettraping and switching rates, Eqs.(27). The factor $X$ is only weakly dependent on experimental parameters and in the first approximation can be considered constant (or even neglected). For the case of S-2DEG-S $\# 2 b, X \simeq 3$. Red dashed lines in Fig. 19 b) represent $I_{R \max }(T)$ calculated from Eq.(24) with $Y=24$ and $X=3$, which perfectly reproduce the simulated $I_{R \max }(T)$ for both $Q_{0}$ values.

Knowing $I_{S \max }(T)$ and $I_{R \max }(T)$, we can easily obtain $T^{*}$ from the condition (cf. Figs. $19 \mathrm{~b}$ and c): 


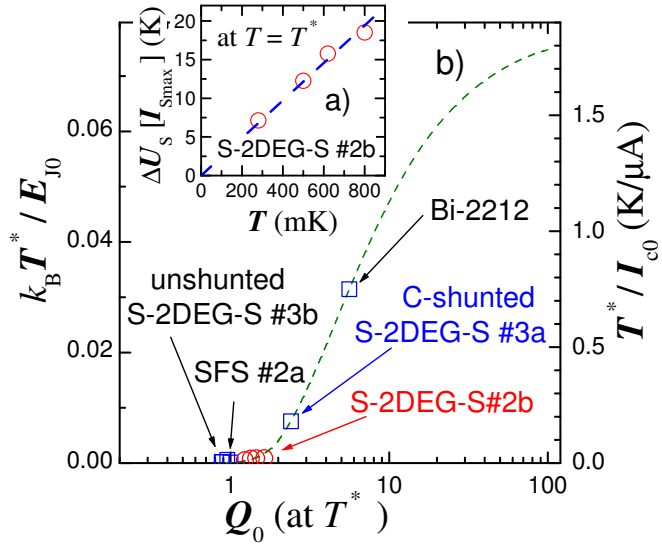

FIG. 20: (Color online). a) The height of the escape barrier at the most probable switching current as a function of $T$ (at $\left.T=T^{*}\right)$ : symbols represent experimental data for S-2DEG-S \#2b from Fig. 15 a), the solid line corresponds to Eq.(22). b) The normalized collapse temperature vs. the quality factor: dashed line represents numerical solution of Eq.(25), symbols represent experimental data for different JJ's.

$$
I_{R \max }\left(T^{*}\right)=I_{\operatorname{Smax}}\left(T^{*}\right) .
$$

A simple analytic estimation of $T^{*}$ can be obtained by observing that $I_{S \max }(T)$ is almost linear in a wide $T$-range, as seen from Figs. $19 \mathrm{~b}$ ) and 11 . In this case Eq.(23) can be approximated as

$$
I_{S \max } / I_{c 0}^{*} \simeq 1-\beta T
$$

where $\beta=2 /\left(3 T_{J}^{2 / 3} T_{0}^{1 / 3}-T_{0}\right), \quad I_{c 0}^{*}=I_{c 0}[1-$ $\left.\left(T_{0} / T_{J}\right)^{2 / 3} / 3\right]$ and $T_{0}$ is some characteristic temperature $\sim T_{J}$. Substituting Eq.(26) into Eq. (25), taking a simple approximation for $I_{R 0}$, Eq.(7), and neglecting $T$-dependence of $I_{c 0}$ we obtain a quadratic equation for $T^{*}$, which yields:

$T^{*} \simeq \frac{k_{B}(Y+X)}{2 \beta^{2} E_{J 0} Q_{0}^{2}}\left[\sqrt{1+\left(\frac{I_{c 0}^{*}}{I_{c 0}}-\frac{4}{\pi Q_{0}}\right) \frac{2 Q_{0}^{2} \beta E_{J 0}}{k_{B}(Y+X)}}-1\right]^{2}$.

From Eq.(27) it follows that $k_{B} T^{*} / E_{J 0}$ strongly depends on $Q_{0}$, but is independent of $E_{J 0}$ because $T$ appear in Eqs. (20124) only in combination $T / E_{J 0}$ (in the case of Eq. (27) because $\left.\beta \sim 1 / E_{J 0}\right)$.

The dashed line in Fig. $20 \mathrm{~b}$ ) represents the numerically simulated $T^{*}$ normalized by $E_{J 0}$ and $I_{c 0}$, (left and right axes, respectively) as a function of the quality factor $Q_{0}$. It was obtained by numerical solution of Eq.(25), without simplifications used for derivation of Eq.(27). It is seen that for overdamped JJ's, $Q_{0}<0.84, T^{*} / I_{c 0} \rightarrow 0$. The $T^{*} / I_{c 0}$ continuously grows with increasing $Q_{0}>0.84$ and saturates at $\sim 2 K / \mu A$ for strongly underdamped JJ's $Q_{0} \gg 1$.

The symbols in Fig.20 b) represent experimental values of $T^{*} / I_{c 0}$ for the JJ's studied in this work. The experimental data agrees well with the proposed theory (dashed line). The simulated values of $T^{*} / I_{c 0}$ are also consistent with experimental data for underdamped SIS-type JJ's $\mathrm{Al}-\mathrm{AlO}_{x}-\mathrm{Al}, T^{*} / I_{c 0} \simeq 1-3.3 K / \mu A$ [1], and $\mathrm{Nb}-\mathrm{AlO}_{x^{-}}$ $\mathrm{Nb}, T^{*} / I_{c 0} \simeq 1 K / \mu A[3]$ (with a reservation that those measurements were done on SQUID's, which may have different activation energies than single JJ's, Eqs.(318).

The $T^{*} / I_{c 0}$ dependence, shown in Fig. $20 \mathrm{~b}$ ), is almost universal and explains the paradoxical collapse of thermal fluctuations, reported in Refs. [1, 2, 3] , as well as the new data presented here. For example, recovery of conventional TA-switching in $C$-shunted S-2DEG-S junctions, see Fig. ??, is caused by the increase of the $Q_{0}$, which according to Fig. $20 \mathrm{~b}$ ) result in larger $T^{*}$ for the same $I_{c 0}$. Similarly, the decrease of $Q_{0}$ due to suppression of $I_{c 0}$ causes the collapse of TA vs. $V_{g}$ in Fig. 14 and the decrease of $T^{*}$ with $H$ in Fig. 15.

From Fig. 20 b) it is seen that in overdamped junctions $T^{*}=0$, implying that retrapping is crucially affecting switching statistics at any $T$. In this case $T_{\text {esc }}(T)$ and $\Delta I(T)$ decrease at all $T$. We observed such behavior for S-2DEG-S junctions with small $I_{c 0}$, and consequently small $Q_{0}$. The tendency of decreasing $T^{*}$ with $I_{c 0}$ is apparent from Fig 15 a).

Therefore, observation of the collapse, i.e., a maximum of $T_{\text {esc }}(T)$ at $T^{*}>0$, is the most unambiguous indication of underdamped, $Q_{0}>0.84$, state in the studied JJ's. The estimation of $Q_{0}$ from the value of $T^{*}$ confirms our assessment of junction capacitances, made in sec. IV.

\section{Phase dynamics in the collapsed state}

The insight into the phase dynamics at $T>T^{*}$ can be obtained from Fig. 20 a), in which the dependence of $\Delta U_{S}\left(I=I_{S \max }\right)$ vs. $T$ is shown for the case of Fig. 15 a). The dashed line corresponds to $\Delta U_{S}\left(I_{S \max }\right) / k_{B} T=$ $24.3 \simeq Y$ obtained from simulations presented in Fig. 19 and demonstrates excellent agreement with the experiment. The large value of $\Delta U_{S} / k_{B} T$ implies that the JJ can escape from the $S$ to the $R$ state only a few times during the time of the experiment. Therefore, the collapse is not due to transition into the phase-diffusion state, which may also lead to reduction of $\Delta I$ [14]. Indeed, phase diffusion requires repeated escape and retrapping, which is only possible for $\Delta U_{S} / k_{B} T \sim 1$ [9, 53]. Careful measurements of supercurrent branches in the IVC's at $T \gtrsim T^{*}$ did not reveal any dc-voltage down to $\sim 10 \mathrm{nV}$ for S-2DEG-S JJ's and $\sim 1 \mu V$ for IJJ's. Furthermore, the IVC's remain hysteretic at $T>T^{*}$, which is incompatible with the phase diffusion within the RCSJ model 9]. As can be seen from Fig. 10, the phase diffusion 
in IJJ's appears only at $T>90 K$, meaning that all the collapse of TA shown in Fig. $13 \mathrm{~b}$ ) at $75 K<T<85 K$ occurs before entering into the phase diffusion state.

Therefore, in the collapse state the junction makes a few very short excursions from the $S$ to the $R$ state during the current sweep, before it eventually switches into the $R$ state. However, the number of excursions and the total excursion time is so small that it does not lead to a measurable dc-voltage in the JJ. The occurrence of the corresponding phase dynamic state, prior to the phasediffusion, has been observed by numerical modelling and discussed in Ref. [9].

\section{CONCLUSIONS}

We have analyzed the influence of damping on the switching current statistics of moderately damped Josephson junctions, employing a variety of methods for accurate tuning of the damping parameter. A paradoxical collapse of switching current fluctuations with increasing temperature was observed in various types of Josephson junctions [1, 2, 3], including low- $T_{c}$ SNS, SFS, S-2DEG-S, SIS, and high- $T_{c}$ intrinsic Josephson junctions. The unusual phenomenon was explained by an interplay of two conflicting consequences of thermal fluctuations, which on the one hand assist in premature switching to the $R$-state and on the other hand help in retrapping back into the $S$-state. In this case the probability of measuring a switching event becomes a conditional probability of switching and not being retrapped during the time of the experiment. Numerical calculations has shown that this model provides a quantitative explanation of both the value of the collapse temperature $T^{*}$, and the unusual shape of switching histograms in the collapsed state. Based on the theoretical analysis, we conclude that the collapse represents a very general phenomenon, which must occur in any underdamped JJ at sufficiently high $T$.

The collapse of switching current fluctuations in Josephson junctions represents an exception from the law of increasing of thermal fluctuations with temperature. In the studied case, the "failure" of this general law of nature, is caused by coexistence of two counteracting processes (switching and retrapping). It should be emphasized that fluctuations for each of the two processes alone follow the law and enhance with $T$ in a conventional manner. It is, however, remarkable and unusual that fluctuations may cancel each other and lead to reduction of thermal fluctuations of a physically measurable quantity.

Finally, we note that the reduced width of switching histograms in the collapsed state of moderately damped JJ's may be advantageous for single-shot read-out of superconducting qubits, which requires accurate discrimination of two close current states.

\section{ACKNOWLEDGMENTS}

We are grateful to S.Intiso, E.Hürfeld, H.Frederiksen, I.Zogaj, A.Yurgens, V.A.Oboznov and V.V.Ryazanov for assistance with sample fabrications and/or measurements; to T.Akazaki and H.Takayanagi for providing S2DEG-S samples; and to R.Gross for lending the sampleand-hold equipment.

[1] J.M.Kivioja, T.E.Nieminen, J.Claudon, O.Buisson, F.W.J.Hekking, and J.P.Pekola, Phys.Rev.Lett. 94, $247002(2005)$

[2] V.M.Krasnov, T.Bauch, S.Intiso, E.Hürfeld, T.Akazaki, H.Takayanagi, and P.Delsing, Phys.Rev.Lett 95, 157002 (2005)

[3] J.Männik, S.Li, W.Qiu, W.Chen, V.Patel, S.Han, and J.E.Lukens, Phys.Rev.B 71, 220509(R) (2005)

[4] P.Hänggi, P.Talkner, and M.Borkovec, Rev.Mod.Phys. 62, 251 (1990)

[5] A.O.Caldeira and A.J.Leggett , Phys.Rev.Lett. 46211 (1981)

[6] E.Ben-Jacob, D.J.Bergman, B.J.Matkowsky, and Z.Schuss, Phys.Rev.A 262805 (1982)

[7] H.Grabert, P.Olschowski and U.Weiss, Phys.Rev.B 36, 1931 (1987)

[8] J.M.Martinis and H.Grabert,Phys.Rev.B 38, 2371 (1988)

[9] R.L.Kautz and J.M.Martinis, Phys.Rev.B 42, 9903 (1990)

[10] S.Washburn,R.A.Webb, R.F.Voss and S.M.Faris, Phys.Rev.Lett. 542712 (1985)

[11] J.M.Martinis, M.H.Devoret, and J.Clarke, Phys.Rev.B 35, 4682 (1987)

[12] P.Silvestrini, S.Pagano, R.Cristiano, O.Liengme and K.E.Gray, Phys.Rev.Lett 60, 844 (1988)

[13] E.Turlot, D.Esteve, C.Urbina, J.M.Martinis, M.H.Devoret, S.Linkwitz, and H.Grabert, Phys.Rev.Lett. 621788 (1989)

[14] D.Vion, M.Gotz, P.Joyez, D.Esteve, M.H.Devoret, Phys.Rev.Lett. 773435 (1996).

[15] M.G.Castellano, et al., J.Appl.Phys. 80, 2922 (1996); ibid., 86, 6405 (1999).

[16] M.Schlosshauer Rev.Mod.Phys. 761267 (2004).

[17] R.McDermott, et al., Science 307, 1299 (2005); A.J. Berkley, et al., ibid. 3001548 (2003).

[18] I.Chiorescu, et al., Nature 431159 (2004).

[19] D.Vion, et al., Science 296886 (2002); Yu.A.Pashkin, et al., Nature 421823 (2003);

[20] T.Bauch, T.Lindström, F.Tafuri, G.Rotoli, P.Delsing, T.Claeson, and F.Lombardi, Science 311, 57 (2006)

[21] K.K.Likharev, "Dynamics of Josephson junctions and circuits" (Gordon \& Breach 1991)

[22] A.A.Golubov, M.Yu.Kupriyanov and E.Ilichev, Rev. Mod. Phys. 76, 411 (2004); T.Bauch, F.Lombardi, F.Tafuri, A.Barone, G.Rotoli, P.Delsing and T.Claeson, Phys.Rev.Lett. 94, 087003 (2005)

[23] NbCuNi bilayers were made by V.A.Oboznov at the Institute of Solid State Physics, Chernogolovka, Russia.

[24] V.M.Krasnov, O.Eriksson, S.Intiso, P.Delsing, V.A.Oboznov, A.S.Prokofiev and V.V.Ryazanov, 
Physica C 418, 16 (2005)

[25] $\mathrm{Nb} / \mathrm{Pt} / \mathrm{Nb}$ trilayers were made by H.Frederiksen at Chalmers University of Technology, Gothenburg, Sweden.

[26] T.Golod, "Nanoscale SFS Josephson junctions made by 3D-FIB sculpturing", Master of Science thesis, Chalmers University of Technology, (May 2005).

[27] S-2DEG-S junctions were provided by T.Akazaki and H.Takayanagi from the NTT Basic Research Laboratories, Kanagawa, Japan.

[28] H.Takayanagi, T.Akazaki, and J.Nitta, Phys.Rev.Lett. 75, 3533 (1995); T.Akazaki, H.Nakano, J.Nitta, and H.Takayanagi, Appl.Phys.Lett. 86, 132505 (2005)

[29] T.Bauch, E.Hürfeld, V.M.Krasnov, P.Delsing, H.Takayanagi, and T.Akazaki, Phys. Rev. B 71, $174502(2005)$

[30] R.Kleiner and P.Müller, Phys.Rev.B 49, 1327 (1994)

[31] V.M.Krasnov, A.Yurgens, D.Winkler, P.Delsing, and T.Claeson, Phys.Rev.Lett. 84, 5860 (2000); V.M.Krasnov, A.E.Kovalev, A.Yurgens, and D.Winkler ibid. 86, 2657 (2001)

[32] V.M.Krasnov, Phys.Rev.B. 65, 140504(R) (2002)

[33] V.M.Krasnov, N.Mros, A.Yurgens, and D.Winkler, Phys.Rev.B 59, 8463 (1999);

[34] A.Irie, Y.Hirari, and G.Oya, Appl.Phys.Lett. 72, 2159 (1998); Yu.I.Latyshev, A.E.Koshelev, V.N.Pavlenko, M.B.Gaifullin, T.Yamashita, and Y.Matsuda, Physica C 367, 365 (2002); S.M.Kim, et.al., Phys.Rev.B 72, 140504(R) (2005)

[35] K.Inomata, et al., Phys.Rev.Lett. 95, 107005 (2005); X.Y.Jin, J.Lisenfeld, Y.Koval, A.Lukashenko, A.V.Ustinov and P.Müller, ibid. 96, 177003 (2006)

[36] V.M. Krasnov, T.Bauch and P.Delsing, Phys.Rev.B 72, 012512 (2005).

[37] The studied Bi-2212 structure had a triple-mesa configuration, containing two contact mesas on top of a common pedestal. This allowed four-probe measurement of IJJ's in the pedestal mesa, shown in Fig. 10. The switching statistics in Figs. 11, 13 and 17 was obtained from a stable switching of one of the IJJ's from the contact mesa. Since contact mesas had approximately two times smaller area than the pedestal mesa, IJJ's in the contact mesa had approximately two times smaller critical current, as seen from comparison of Figs. 10 and 11. More details about triple mesa structures can be found in V.M.Krasnov, Phys.Rev.Lett. 97, 257003 (2006).

[38] A.T.Johnson, C.J.Lobb, and M.Tinkham, Phys.Rev.Lett. 65, 1263 (1990).

[39] T.A. Fulton and L.N.Dunkleberger, J.Appl.Phys. 45, 2283 (1974); W.J.Skocpol', M.R.Beasley, and M.Tinkham, ibid 45, 4054 (1974).

[40] Y. Song, J.Appl.Phys. 47, 2651 (1976)

[41] V.M.Krasnov, M.Sandberg and I.Zogaj, Phys.Rev.Lett. 94, 077003 (2005)

[42] V.M.Krasnov, V.A.Oboznov and V.V.Ryazanov, Physica C 196, 335 (1992)

[43] V.M.Krasnov, N.F.Pedersen, V.A.Oboznov, and V.V.Ryazanov Phys.Rev.B 49, 12969 (1994)

[44] V.M.Krasnov, N.F.Pedersen, and V.A.Oboznov, Phys.Rev.B 50, 1106 (1994)

[45] I.Zutic, J.Fabian, and S.Das Sarma, Rev.Mod.Phys. 76, 323 (2004).

[46] A minor decrease of resistance of $C$-shunted S-2DEG-S JJ's is probably associated with the removal of the Algate electrode from the top of the semiconducting heterostructure during the $C$-shunt fabrication. This removes the Shotkey barrier, present below the gate electrode in the unshunted JJ.

[47] B.Bayraktaroglu, J.Barrette, L.Kehias, C.I.Huang, R.Fitch, R.Neidhard, and R.Scherer, IEEE Trans. Electron. Device Lett. 14, 493 (1993)

[48] V.M.Krasnov, A.Yurgens, D.Winkler and P.Delsing, J. Appl. Phys. 89, 5578 (2001); ibid., 93, 1329 (2003).

[49] V.M.Krasnov, V.A.Oboznov, V.V.Ryazanov, N.Mros, A.Yurgens, and D.Winkler, Phys. Rev. B 61, 766 (2000).

[50] P.A.Warburton, et al., J.Appl.Phys. 95, 4941 (2004)

[51] N.Mros, V.M.Krasnov, A.Yurgens, D.Winkler, and T.Claeson, Phys.Rev.B 57 R8135 (1998)

[52] V.M.Krasnov and D.Winkler, Phys.Rev.B 56, 9106 (1997)

[53] A.Franz, et al., Phys.Rev.B 69, 014506 (2004). 